\title{
A Semianalytic Method for Vibration Analysis of a Sandwich FGP Doubly Curved Shell with Arbitrary Boundary Conditions
}

\author{
Zhongyu Zhang $\mathbb{D}^{1},{ }^{1}$ Jiayang Gu $\mathbb{D}^{1},{ }^{1}$ Jianjun Ding, ${ }^{2}$ and Yanwu Tao ${ }^{1}$ \\ ${ }^{1}$ Marine Equipment and Technology Institute, Jiangsu University of Science and Technology, Zhenjiang 212003, Jiangsu, China \\ ${ }^{2}$ Shanghai Marine Diesel Engine Research Institute, Shanghai 200090, China \\ Correspondence should be addressed to Jiayang Gu; gujiayang@126.com
}

Received 30 April 2021; Revised 19 October 2021; Accepted 3 November 2021; Published 7 December 2021

Academic Editor: Jie Yang

Copyright (c) 2021 Zhongyu Zhang et al. This is an open access article distributed under the Creative Commons Attribution License, which permits unrestricted use, distribution, and reproduction in any medium, provided the original work is properly cited.

\begin{abstract}
Due to the excellent mechanical properties of doubly curved structure and functionally graded porous (FGP) material, the study of their vibration characteristics has attracted wide attention. The main aim of this research is to establish a formulation for free and forced vibration analysis of a new Sandwich FGP doubly curved structure. Four models of Sandwich materials are considered. The potential energy and kinetic energy functions are obtained on the foundation of the first-order shear deformation theory (FSDT). The idea of domain energy decomposition is applied to the theoretical modeling, where the structure is segmented along the generatrix direction. The continuity conditions for the interfaces between adjacent segments are balanced by the weighted parameters. For each segment, the displacement functions are selected as the Jacobi orthogonal polynomials and trigonometric series. The boundary conditions of the structure are obtained by the boundary spring simulation technique. The solution is obtained by the variational operation of the structural functional. The convergence performance and correctness of the theoretical model are examined by several numerical examples. Finally, some novel results are given, where free and forced vibration characteristics of Sandwich FGP doubly curved structures are examined in detail.
\end{abstract}

\section{Introduction}

The doubly curved structure is widely used in the construction industry because of its excellent structural and mechanical properties [1-5]. Due to the appearance of pore, functionally graded porous (FGP) materials have better mechanical properties than traditional functionally graded materials [6-10]. Sandwich structures, which own outstanding mechanical properties, have been extensively applied in aerospace, transportation, and other fields [11-15]. However, the Sandwich structure combined with FGP materials has not been investigated yet. The objective of the paper is to establish a model for free and forced vibration analysis of a new Sandwich FGP doubly curved structure based on the semianalytical method.

Zenkour [16, 17] conducted the bending and free vibration analysis of functionally graded (FG) ceramic- metal Sandwich plates by proposing a two-dimensional solution. It was assumed that the faces of structure are with isotropic two-constituent material distribution along the thickness direction, and their elasticity modulus and Poisson's ratio vary in terms of a power-law distribution, while the core layer is homogeneous and consisted of isotropic ceramic material. Natarajan and Manickam [18] studied the bending and free flexural vibration behavior of Sandwich functionally graded material (FGM) plates by using QUAD-8 shear flexible element. Mahi et al. [19] presented a new hyperbolic shear deformation theory to conduct the bending and free vibration analysis of isotropic, FG, Sandwich, and laminated composite plates. Extending the Moving Krigingbased mesh-free method, Thai et al. [20] surveyed the static, dynamic, and buckling analysis of FG isotropic and Sandwich plates with classical boundary conditions. Xiao 
et al. [21] applied the finite element method to study the vibration behavior of Sandwich panel with mass density gradient (DG) foam core on the basis of the high-order Sandwich plate theory. Liu et al. [22] investigated the free vibration characteristics of FG material Sandwich plates based on the refined higher order Sandwich panel theory. Zenkour [23] proposed a quasi-3D shear deformation theory to study the bending responses of FG singlelayered and Sandwich plates with porosities by using Navier's technique. Using a layer-wise finite element method, Pandey and Pradyumna [24] studied the free vibration of FG Sandwich shells in the framework of FSDT. Chen et al. [25] conducted vibration investigation of FGM Sandwich doubly curved shallow shell by a novel shear deformation theory and the Navier method. Trinh and Kim [26] proposed a closed-form solution to study the nonlinear characteristics of the FG Sandwich shells with thermomechanical loadings.

A retrospect of previous researches shows that most of the investigations were focusing on Sandwich FGM structures, while FGP materials were rarely studied. In addition, the Sandwich rectangular plate or cylindrical shell has been sufficiently examined, while the research on Sandwich doubly curved structure is scarce.

This paper aims to construct a vibration analysis model for Sandwich FGP doubly curved shell subjected to arbitrary boundary conditions based on the FSDT, where four Sandwich material models are considered. The domain energy decomposition technique is employed, which can make the selection of displacement functions become rather flexible. The weighted parameters are utilized to deal with the continuity conditions between each neighbored segment. For every segment, the displacement functions are represented by Jacobi polynomials along the generatrix orientation and trigonometric series in the circumferential orientation. Free vibration and forced response can be obtained by standard variational operation. The convergence and validity of the model are shown by several numerical cases.

\section{Theoretical Formulations}

2.1. Geometric Description. The orthogonal coordinate system $(\varphi, \theta, z)$ of the Sandwich FGP doubly curved shell is shown in Figure 1, where $o z$ and $o^{\prime} z^{\prime}$ are the spatial coordinate axis and geometric central axis, respectively. By spinning the generatrix with respect to the central axis $o^{\prime} z^{\prime}$, structural surface can be achieved. $R_{\varphi}$ and $R_{\theta}$ are primary curvature radius, the centers of which are $O_{\varphi}$ and $O_{\theta}$ respectively. $R_{s}$ represents the offset distance between $o^{\prime} z^{\prime}$ and $o z . R_{0}$ signifies the sum of horizontal radius and $R_{s}$, where $R_{0}=R_{\theta} \sin \varphi$. In this paper, three kinds of doubly curved shells are studied, and the corresponding generatrix characteristics are shown in Figure 2. The specific geometric equations are expressed as follows $[27,28]^{:}$

(1) Elliptical shell (Figure 2(a)):

$$
\begin{aligned}
& R_{\varphi}(\varphi)=\frac{a^{2} b^{2}}{\sqrt{\left(a^{2} \sin ^{2} \varphi+b^{2} \cos ^{2} \varphi\right)^{3}}} \\
& R_{\theta}(\varphi)=\frac{a^{2}}{\sqrt{a^{2} \sin ^{2} \varphi+b^{2} \cos ^{2} \varphi}}+\frac{R_{s}}{\sin \varphi}
\end{aligned}
$$

where $a$ indicates the length of the semimajor axes, and $b$ represents that of the semiminor axes.

$$
\begin{aligned}
b & =\left(\frac{a L}{\left(\sqrt{a^{2}-R_{0}^{2}}-\sqrt{a^{2}-R_{1}^{2}}\right)}\right) \\
\varphi_{0} & =\arctan \left(\frac{b R_{0}}{a \sqrt{a^{2}-R_{0}^{2}}}\right), \\
\varphi_{1} & =\arctan \left(\frac{b R_{1}}{a \sqrt{a^{2}-R_{1}^{2}}}\right) .
\end{aligned}
$$

(2) Paraboloidal shell (Figure 2(b)):

$$
\begin{aligned}
& R_{\varphi}(\varphi)=\frac{k}{2 \cos ^{3} \varphi}, \\
& R_{\theta}(\varphi)=\frac{k}{2 \cos \varphi}+\frac{R_{s}}{\sin \varphi},
\end{aligned}
$$

where

$$
\begin{gathered}
k=\frac{R_{1}^{2}-R_{0}^{2}}{L} \\
\varphi_{0}=\arctan \left(\frac{2 R_{0}}{k}\right), \\
\varphi_{1}=\arctan \left(\frac{2 R_{1}}{k}\right) .
\end{gathered}
$$

(3) Hyperbolic shell (Figure 2(c)):

$$
\begin{aligned}
& R_{\varphi}(\varphi)=\frac{-a^{2} b^{2}}{\sqrt{\left(a^{2} \sin ^{2} \varphi-b^{2} \cos ^{2} \varphi\right)^{3}}}, \\
& R_{\theta}(\varphi)=\frac{a^{2}}{\sqrt{a^{2} \sin ^{2} \varphi-b^{2} \cos ^{2} \varphi}}+\frac{R_{s}}{\sin \varphi} .
\end{aligned}
$$

Specially,

$$
\begin{aligned}
b & =a c / \sqrt{R_{0}^{2}-a^{2}}=a D / \sqrt{R_{1}^{2}-a^{2}}, \\
\varphi_{0} & =\arctan \left(\frac{b R_{0}}{a \sqrt{R_{0}^{2}-a^{2}}}\right), \\
\varphi_{1} & =\pi-\arctan \left(\frac{b R_{1}}{a \sqrt{R_{1}^{2}-a^{2}}}\right) .
\end{aligned}
$$



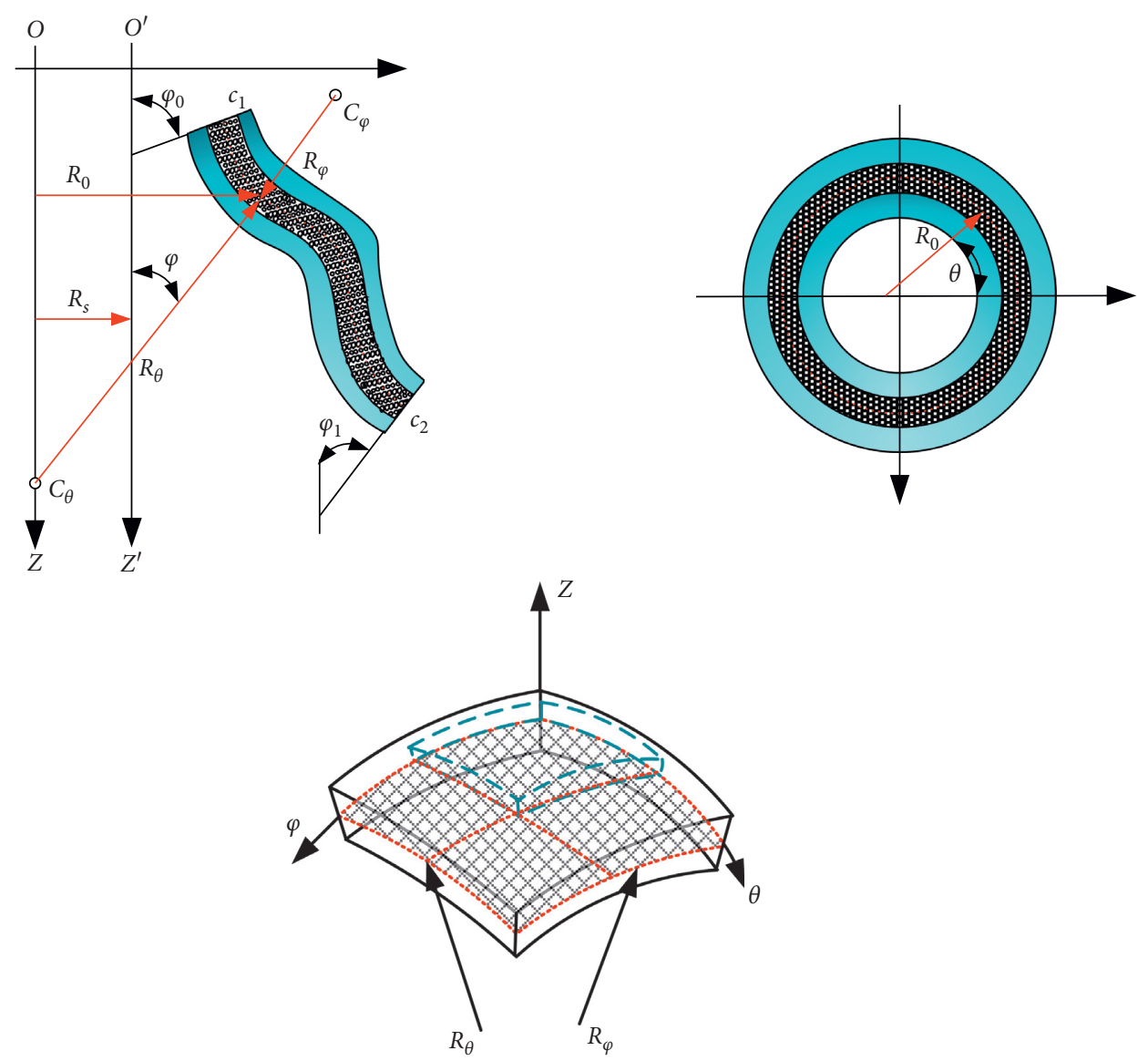

Figure 1: Geometric and reference system of a Sandwich FGP doubly curved structure.
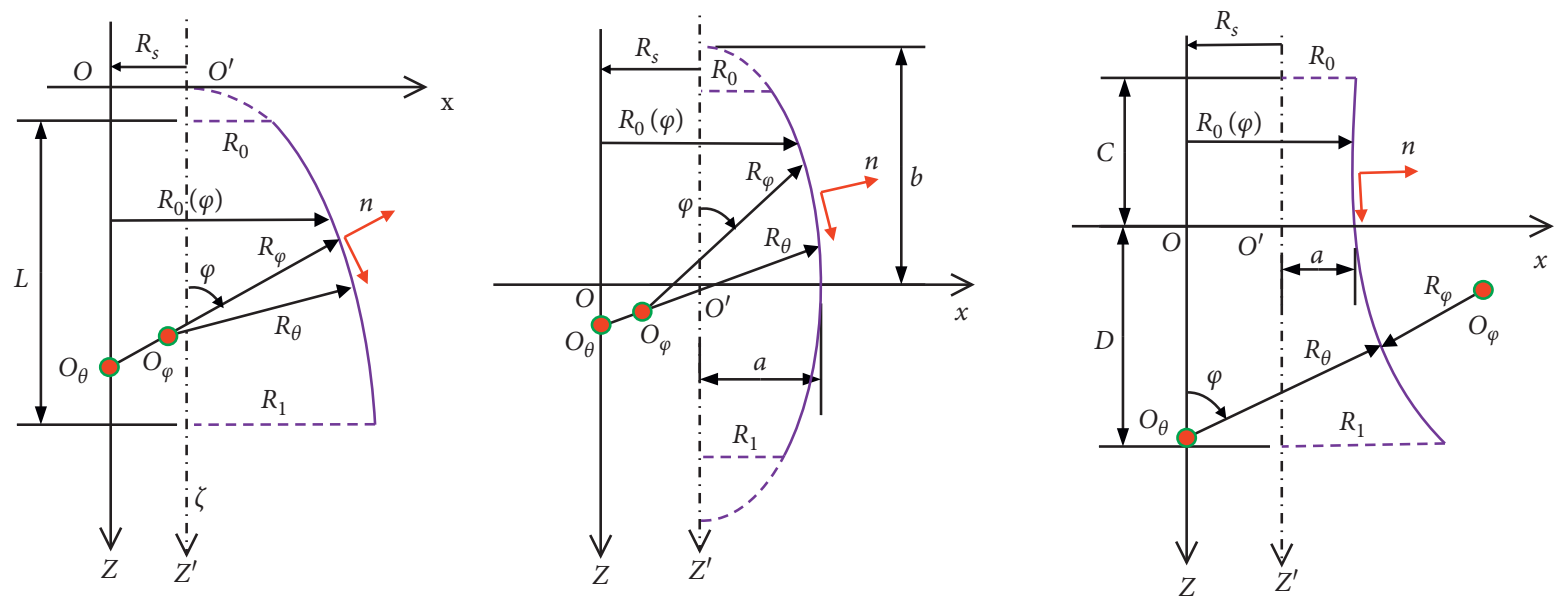

FIGURE 2: Generatrix diagram of three Sandwich FGP doubly curved structures.

2.2. Material Model. For FGP materials, there are three kinds of commonly used mechanical models: symmetric distribution model, nonsymmetric distribution model, and uniform distribution model [29]. In this paper, symmetric distribution model and nonsymmetric distribution model are considered. Four types of Sandwich FGP materials are shown in Figure 3. The material properties along the thickness direction are expressed as follows:

$$
\begin{aligned}
& E(z)=E_{1}\left(1-e_{0} \alpha\right), \\
& G(z)=G_{1}\left(1-e_{0} \alpha\right),
\end{aligned}
$$






Type1-1

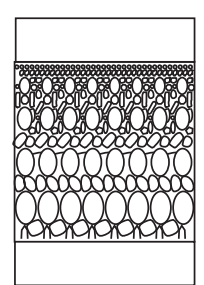

Type1-2



Type2-1

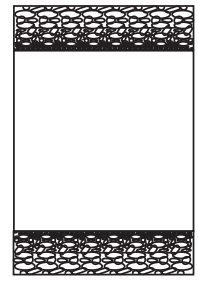

Type2-2
FIGURE 3: Schematic diagrams of four types of Sandwich FGP materials.

$$
\rho(z)=\rho_{1}\left(1-e_{m} \alpha\right)
$$

The expressions of pore characteristic coefficients $e_{0}$ and $e_{m}$ are

$$
\begin{aligned}
& e_{0}=1-\frac{E_{2}}{E_{1}}=1-\frac{G_{2}}{G_{1}}, \quad 0 \leq e_{0} \leq 1, \\
& e_{m}=1-\frac{\rho_{2}}{\rho_{1}}, \quad 0 \leq e_{m} \leq 1, \\
& e_{m}=1-\sqrt{1-e_{0}} .
\end{aligned}
$$

It is obvious from equation (4) that, for different types of Sandwich FGP materials, only material parameter $\alpha$ can be adjusted. The expressions of material parameters $\alpha$ are as follows:

$$
\begin{aligned}
& \alpha= \begin{cases}0 & z \in\left[z_{1}, z_{2}\right] \\
\cos \left(\frac{\pi\left(z-z_{i}\right)}{\left(z_{i+1}-z_{i}\right)}-\frac{\pi}{2}\right) & z \in\left[z_{2}, z_{3}\right], \\
0 & z \in\left[z_{3}, z_{4}\right]\end{cases} \\
& \alpha= \begin{cases}1 & z \in\left[z_{1}, z_{2}\right] \\
\cos \left(\frac{\pi\left(z-z_{i}\right)}{2\left(z_{i+1}-z_{i}\right)}\right) & z \in\left[z_{2}, z_{3}\right], \\
0 & z \in\left[z_{3}, z_{4}\right]\end{cases} \\
& \alpha= \begin{cases}\cos \left(\frac{\pi\left(z-z_{i}\right)}{\left(z_{i+1}-z_{i}\right)}-\frac{\pi}{2}\right) & z \in\left[z_{1}, z_{2}\right] \\
0 & z \in\left[z_{2}, z_{3}\right], \\
\cos \left(\frac{\pi\left(z-z_{i}\right)}{\left(z_{i+1}-z_{i}\right)}-\frac{\pi}{2}\right) & z \in\left[z_{3}, z_{4}\right]\end{cases}
\end{aligned}
$$

$$
\alpha= \begin{cases}\cos \left(\frac{\pi\left(z-z_{i}\right)}{2\left(z_{i+1}-z_{i}\right)}\right) & z \in\left[z_{1}, z_{2}\right] \\ 0 & z \in\left[z_{2}, z_{3}\right] \\ 1-\cos \left(\frac{\pi\left(z-z_{i}\right)}{2\left(z_{i+1}-z_{i}\right)}\right) & z \in\left[z_{3}, z_{4}\right]\end{cases}
$$

2.3. Energy Functional of Shell Segment. In this paper, the FSDT is applied in the process of modeling [30, 31]. The displacement fields of Sandwich FGP doubly curved shells are

$$
\begin{aligned}
& \bar{U}(\varphi, \theta, z, t)=u(\varphi, \theta, t)+z \psi_{\varphi}(\varphi, \theta, t), \\
& \bar{V}(\varphi, \theta, z, t)=v(\varphi, \theta, t)+z \psi_{\theta}(\varphi, \theta, t), \\
& \bar{W}(\varphi, \theta, z, t)=w(\varphi, \theta, t),
\end{aligned}
$$

where $u, \mathrm{v}$ and $w$ are displacements in the $\varphi, \theta$ and $z$ directions on the middle surface, and $\psi_{\varphi}$ and $\psi_{\theta}$ are rotations of the normal with respect to the $\theta$ and $\varphi$ orientations. The linear strain-displacement relationships can be shown as

$$
\begin{aligned}
& \varepsilon_{\varphi}^{0}=\frac{1}{A} \frac{\partial u}{\partial \varphi}+\frac{v}{A B} \frac{\partial A}{\partial \theta}+\frac{w}{R_{\varphi}}, \\
& \varepsilon_{\theta}^{0}=\frac{1}{B} \frac{\partial v}{\partial \theta}+\frac{u}{A B} \frac{\partial B}{\partial \varphi}+\frac{w}{R_{\theta}}, \\
& \gamma_{\varphi \theta}^{0}=\frac{A}{B} \frac{\partial}{\partial \theta}\left(\frac{u}{A}\right)+\frac{B}{A} \frac{\partial}{\partial \varphi}\left(\frac{v}{B}\right), \\
& k_{\varphi}=\frac{1}{A} \frac{\partial \psi_{\varphi}}{\partial \varphi}+\frac{\psi_{\theta}}{A B} \frac{\partial A}{\partial \theta}, \\
& k_{\theta}=\frac{1}{B} \frac{\partial \psi_{\theta}}{\partial \theta}+\frac{\psi_{\varphi}}{A B} \frac{\partial B}{\partial \varphi}, \\
& k_{\varphi \theta}=\frac{A}{B} \frac{\partial}{\partial \theta}\left(\frac{\psi_{\varphi}}{A}\right)+\frac{B}{A} \frac{\partial}{\partial \varphi}\left(\frac{\psi_{\theta}}{B}\right), \\
& \gamma_{\varphi z}^{0}=\frac{1}{A} \frac{\partial w}{\partial \varphi}-\frac{u}{R_{\varphi}}+\psi_{\varphi}, \\
& \gamma_{\theta z}^{0}=\frac{1}{B} \frac{\partial w}{\partial \theta}-\frac{v}{R_{\theta}}+\psi_{\theta}, \\
& A=R_{\varphi}, B=R_{\theta} \sin \varphi .
\end{aligned}
$$

The relations between force/moment resultants and strains/curvatures change are 


$$
\begin{aligned}
& {\left[\begin{array}{c}
N_{\varphi} \\
N_{\theta} \\
N_{\varphi \theta} \\
M_{\varphi} \\
M_{\theta} \\
M_{\varphi \theta}
\end{array}\right]=\left[\begin{array}{cccccc}
A_{11} & A_{12} & 0 & B_{11} & B_{12} & 0 \\
A_{12} & A_{22} & 0 & B_{12} & B_{22} & 0 \\
0 & 0 & A_{66} & 0 & 0 & B_{66} \\
B_{11} & B_{12} & 0 & D_{11} & D_{12} & 0 \\
B_{12} & B_{22} & 0 & D_{12} & D_{22} & 0 \\
0 & 0 & B_{66} & 0 & 0 & D_{66}
\end{array}\right]\left[\begin{array}{c}
\varepsilon_{\varphi} \\
\varepsilon_{\theta} \\
\gamma_{\varphi \theta} \\
k_{\varphi} \\
k_{\theta} \\
k_{\varphi \theta}
\end{array}\right],} \\
& {\left[\begin{array}{c}
Q_{\varphi} \\
Q_{\theta}
\end{array}\right]=\kappa_{\text {shear }}\left[\begin{array}{cc}
A_{66} & 0 \\
0 & A_{66}
\end{array}\right]\left[\begin{array}{c}
\gamma_{\varphi z} \\
\gamma_{\theta z}
\end{array}\right],}
\end{aligned}
$$

where the stiffness coefficients are obtained as follows:

$$
\begin{aligned}
\left\{A_{i j}, B_{i j}, C_{i j}\right\} & =\int_{-h / 2}^{h / 2} Q_{i j}(z)\left(1, z, z^{2}\right) d z \\
Q_{11}(z) & =\frac{E(z)}{1-\mu^{2}(z)}, Q_{12}(z)=\frac{\mu(z) E(z)}{1-\mu^{2}(z)}, Q_{66}(z)=\frac{E(z)}{2[1+\mu(z)]} .
\end{aligned}
$$

In this research, the domain energy decomposition technique is exploited. To this end, the structure is divided into $N$ segments along the axis direction. By introducing penalty parameters, the continuity conditions for the interfaces can be realized. According to the FSDT, the strain energy $U_{i}$ and kinetic energy $T_{i}$ of $i$ th segments can be expressed as

$$
\begin{aligned}
& U_{i}=\frac{1}{2} \iiint_{V}\left(\begin{array}{c}
N_{\varphi} \varepsilon_{\varphi}^{0}+N_{\theta} \varepsilon_{\theta}^{0}+N_{\varphi \theta} \gamma_{\varphi \theta}^{0}+M_{\varphi} k_{\varphi}+ \\
M_{\varphi} k_{\theta}+M_{\varphi \theta} k_{\varphi \theta}+Q_{\varphi} \gamma_{\varphi z}^{0}+Q_{\theta} \gamma_{\theta z}^{0}
\end{array}\right) A B d \varphi d \theta d z, \\
& T_{i}=\frac{1}{2} \iiint_{V}^{\rho} \rho(z)\left[(\dot{\bar{U}})^{2}+(\dot{\bar{V}})^{2}+(\dot{\bar{W}})^{2}\right]\left(1+\frac{z}{R_{\varphi}}\right)\left(1+\frac{z}{R_{\theta}}\right) A B d \varphi d \theta d z \\
& \quad=\frac{1}{2} \int_{\varphi_{0}}^{\varphi_{1}} \int_{0}^{2 \pi}\left\{I_{0}\left[(\dot{u})^{2}+(\dot{v})^{2}+(\dot{w})^{2}\right]+2 I_{1}\left(\dot{u} \dot{\psi}_{\varphi}+\dot{v} \dot{\psi}_{\theta}\right)+I_{2}\left[\left(\dot{\psi}_{\varphi}\right)^{2}+\left(\dot{\psi}_{\theta}\right)^{2}\right]\right\} A B d \varphi d \theta,
\end{aligned}
$$

where

$$
\left(I_{0}, I_{1}, I_{2}\right)=\int_{-h / 2}^{h / 2} \rho(z)\left(1+\frac{z}{R_{\varphi}}\right)\left(1+\frac{z}{R_{\theta}}\right)\left(1, z, z^{2}\right) d z .
$$

As mentioned previously, the boundary spring simulation technique is exploited to simulate the corresponding boundary conditions. In addition, the continuity conditions are simulated by weighted parameters. The corresponding boundary and coupling potential energy can be described as

$$
\begin{aligned}
U_{b}= & \frac{1}{2} \int_{0}^{2 \pi} \int_{-h / 2}^{h / 2}\left\{k_{u, 0} u^{2}+k_{v, 0} v^{2}+k_{w, 0} w^{2}+k_{\varphi, 0} \psi_{\varphi}^{2}+k_{\theta, 0} \psi_{\theta}^{2}\right\}_{\varphi_{0}=0} B d \theta d z \\
& +\frac{1}{2} \int_{0}^{2 \pi} \int_{-h / 2}^{h / 2}\left\{k_{u, 1} u^{2}+k_{v, 1} v^{2}+k_{w, 1} w^{2}+k_{\varphi, 1} \psi_{\varphi}^{2}+k_{\theta, 1} \psi_{\theta}^{2}\right\}_{\varphi_{0}=L} B d \theta d z . \\
U_{c}^{i}= & \frac{1}{2} \int_{0}^{2 \pi} \int_{-h / 2}^{h / 2}\left\{\begin{array}{c}
k_{u}\left(u_{i}-u_{i+1}\right)^{2}+k_{v}\left(v_{i}-v_{i+1}\right)^{2}+k_{w}\left(w_{i}-w_{i+1}\right)^{2} \\
+k_{\varphi}\left(\psi_{\varphi_{i}}-\psi_{\varphi_{i+1}}\right)^{2}+k_{\theta}\left(\psi_{\theta_{i}}-\psi_{\theta_{i+1}}\right)^{2}
\end{array}\right\} B d \theta d z,
\end{aligned}
$$


where $i$ and $i+1$ represent the $i^{\text {th }}$ and $(i+1)^{\text {th }}$ shell segments. When considering forced vibration, the work done by the external loads may be represented as

$$
W_{i}=\frac{1}{2} \int_{\varphi} \int_{\theta}\left(f_{u_{i}} u_{i}+f_{v_{i}} v_{i}+f_{w_{i}} w_{i}+m_{\varphi_{i}} \psi_{\varphi_{i}}+m_{\theta_{i}} \psi_{\theta_{i}}\right) R_{\varphi} R_{0} d \varphi d \theta
$$

where $f_{u_{i}}, f_{v_{i}}$ and $f_{w_{i}}$ signify the related distributed forces along the $\varphi, \theta$ and $z$ orientations, respectively; and the corresponding distributed couples with respect to the reference surface are represented by $m_{\varphi_{i}}$ and $m_{\theta_{i}}$.

As a result, the Lagrangian energy functions $(L)$ can be displayed as

$$
L=\sum_{i}^{N}\left(T_{i}-U_{i}+W_{i}\right)-\sum_{i=1}^{N-1} U_{c, i}-U_{b} .
$$

2.4. Solution Methodology. With regard to the trial function method, the key issue is constructing a displacement admissible function, which satisfies the boundary conditions. In this paper, by introducing the domain energy decomposition technique, the structure is segmented, and weighted parameters are brought in to simulate the continuity conditions for the interfaces between each neighbored segment. This can make the choices of displacement admissible function become quite flexible. Since all segments are free boundaries, only the displacement functions are required to be continuous and orthogonal. Herein, the Jacobian orthogonal polynomials [32-34] are utilized for the expansion of the displacement. The displacement functions are exhibited as follows:

$$
\begin{gathered}
u=\sum_{m=0}^{M} \sum_{n=0}^{N} U_{m n, i} P_{m}^{(\alpha, \beta)}(\varphi)[\cos (n \theta)+\sin (n \theta)] e^{i \omega t}, \\
v=\sum_{m=0}^{M} \sum_{n=0}^{N} V_{m n, i} P_{m}^{(\alpha, \beta)}(\varphi)[\sin (n \theta)+\cos (n \theta)] e^{i \omega t}, \\
w=\sum_{m=0}^{M} \sum_{n=0}^{N} W_{m n, i} P_{m}^{(\alpha, \beta)}(\varphi)[\cos (n \theta)+\sin (n \theta)] e^{i \omega t}, \\
\psi_{\varphi}=\sum_{m=0}^{M} \sum_{n=0}^{N} \psi_{\varphi_{m n, i}} P_{m}^{(\alpha, \beta)}(\varphi)[\cos (n \theta)+\sin (n \theta)] e^{i \omega t}, \\
\phi_{\varphi}=\sum_{m=0}^{M} \sum_{n=0}^{N} \phi_{\varphi_{m n, i}} P_{m}^{(\alpha, \beta)}(\varphi)[\cos (n \theta)+\sin (n \theta)] e^{i \omega t},
\end{gathered}
$$

where $U_{m n, i}, V_{m n, i}, W_{m \eta, i}, \psi_{\varphi_{m n, i}}$ and $\phi_{\varphi_{m n, i}}$ are the Jacobi expanded coefficients; $P_{m}^{(\alpha, \beta)}(\varphi)$ is the Jacobi polynomial of $m$ th order, representing the displacement components along the generatrix orientation. In practice, different types of orthogonal polynomials can be obtained by resetting Jacobian parameters $\alpha$ and $\beta$. It must also be pointed out here that the relative errors between the calculated results under different Jacobian parameters are very small. In this paper, the parameters of Jacobi are selected as $\alpha=\beta=0$ (Legendre polynomials).
Substituting equations (21) into equation (20) and expressing it in the form of partial differential equation, the discretized equation of motion is obtained:

$$
\begin{aligned}
& \frac{\partial L}{\partial q}=0, \\
& q=U_{m n, i}, V_{m n, i}, W_{m n, i}, \psi_{x_{m n, i}}, \phi_{x_{m n, i}} .
\end{aligned}
$$

Then, the forced vibration characteristic equation may be achieved:

$$
\mathbf{M} \ddot{\mathbf{q}}+\left[\mathbf{K}+\mathbf{K}_{C}+\mathbf{K}_{B}\right] \mathbf{q}=\mathbf{F},
$$

where $\mathbf{M}$ denotes the generalized mass matrix; $\mathbf{K}$ indicates the disjoint generalized stiffness matrix; $\mathbf{K}_{C}$ and $\mathbf{K}_{B}$ are the stiffness matrices related with weighted and boundary parameters, respectively. By assuming harmonic motions $\mathbf{q}=\widetilde{q} e^{i \omega t}$, the governing equations can be derived from equation (23):

$$
\operatorname{det}\left[-\omega^{2} \mathbf{M}+\left(\mathbf{K}-\overline{\mathbf{K}}_{\lambda}-\overline{\mathbf{K}}_{\gamma}-\hat{\mathbf{K}}_{B}\right)\right]=0 .
$$

Through the solution of equation (24), the frequencies as well as the mode shapes can be obtained.

\section{Convergence Studies and Numerical Verification}

The accuracy of the whole theory should be closely related to the expansion coefficients of Jacobian orthogonal polynomials, the number of segments, and the weighted parameters between segments. Therefore, it is essential to study the convergence and correctness of the numerical results. In order to simplify the description of edge conditions, the F, C, and $\operatorname{Ei}(i=1,2,3)$ represent the free, clamped, and elastic edge conditions, respectively.

Table 1 shows the convergence analysis of the number of segments for Sandwich FGP doubly curved structure. The material parameters are defined as follows: $E_{1}=70 \mathrm{GPa}$, $\mu=0.3, \rho=2702 \mathrm{~kg} / \mathrm{m}^{3}, e_{0}=0.2$. Sandwich material type is $2-1$. If the material parameters are not specified later, the material parameters will remain unchanged. The thickness ratio coefficient of Sandwich structure is defined as $1: 3: 1$. The geometric parameters are defined as follows: paraboloidal shell: $R_{0}=2 \mathrm{~m}, R_{1}=4 \mathrm{~m}, R_{s}=1 \mathrm{~m}, k=1, h=0.1 \mathrm{~m}$; hyperbolic shell: $R_{1}=2 \mathrm{~m}, R_{s}=1 \mathrm{~m}, c=1 \mathrm{~m}, D=4 \mathrm{~m}$, $h=0.1 \mathrm{~m}$; elliptical shell: $a_{1}=1 \mathrm{~m}, b_{1}=2 \mathrm{~m}, R_{s}=1 \mathrm{~m}$, $h=0.1 m, \varphi_{0}=\pi / 6, \varphi_{0}=5 \pi / 6$. As shown in Table 1 , it is obvious that when the number of segments exceeds 3 , the computed results tend to be stable. Therefore, in all subsequent examples, the number of segments is chosen to be $N=4$. Table 2 shows the convergence analysis of truncation terms for Sandwich FGP doubly curved structure with C-F boundary conditions. The geometric dimensions and material parameters are in accordance with those in Table 1.

From Table 2, it is also found that when the Jacobian orthogonal polynomial is expanded to six terms, the calculated results can be regarded as exact ones. Therefore, in 
TABLE 1: Convergence analysis of number of segments for Sandwich FGP doubly curved structure.

\begin{tabular}{|c|c|c|c|c|c|c|c|c|c|c|c|c|}
\hline \multirow{2}{*}{$N$} & \multicolumn{4}{|c|}{ Paraboloidal shell } & \multicolumn{4}{|c|}{ Hyperbolic shell } & \multicolumn{4}{|c|}{ Elliptical shell } \\
\hline & 1 & 2 & 3 & 4 & 1 & 2 & 3 & 4 & 1 & 2 & 3 & 4 \\
\hline 2 & 9.46 & 9.80 & 15.94 & 23.30 & 42.91 & 47.49 & 56.58 & 74.94 & 45.57 & 60.60 & 128.43 & 167.75 \\
\hline 3 & 9.45 & 9.80 & 15.91 & 23.30 & 42.83 & 47.47 & 56.41 & 74.93 & 45.56 & 60.59 & 128.40 & 167.75 \\
\hline 4 & 9.45 & 9.80 & 15.90 & 23.30 & 42.79 & 47.47 & 56.30 & 74.93 & 45.56 & 60.59 & 128.40 & 167.75 \\
\hline 5 & 9.45 & 9.80 & 15.90 & 23.30 & 42.78 & 47.47 & 56.25 & 74.94 & 45.56 & 60.59 & 128.40 & 167.75 \\
\hline 6 & 9.45 & 9.80 & 15.90 & 23.30 & 42.77 & 47.47 & 56.23 & 74.94 & 45.56 & 60.59 & 128.40 & 167.75 \\
\hline 8 & 9.45 & 9.80 & 15.90 & 23.30 & 42.77 & 47.47 & 56.21 & 74.94 & 45.56 & 60.59 & 128.40 & 167.75 \\
\hline 10 & 9.45 & 9.80 & 15.90 & 23.30 & 42.77 & 47.47 & 56.21 & 74.94 & 45.56 & 60.59 & 128.40 & 167.75 \\
\hline
\end{tabular}

TABLE 2: Convergence analysis of truncation terms for Sandwich FGP doubly curved structure.

\begin{tabular}{|c|c|c|c|c|c|c|c|c|c|c|c|c|}
\hline \multirow{2}{*}{$m$} & \multicolumn{4}{|c|}{ Paraboloidal shell } & \multicolumn{4}{|c|}{ Hyperbolic shell } & \multicolumn{4}{|c|}{ Elliptical shell } \\
\hline & 1 & 2 & 3 & 4 & 1 & 2 & 3 & 4 & 1 & 2 & 3 & 4 \\
\hline 2 & 9.46 & 9.80 & 15.94 & 23.30 & 42.91 & 47.49 & 56.58 & 74.94 & 45.57 & 60.60 & 128.43 & 167.75 \\
\hline 3 & 9.45 & 9.80 & 15.91 & 23.30 & 42.83 & 47.47 & 56.41 & 74.93 & 45.56 & 60.59 & 128.40 & 167.75 \\
\hline 4 & 9.45 & 9.80 & 15.90 & 23.30 & 42.79 & 47.47 & 56.30 & 74.93 & 45.56 & 60.59 & 128.40 & 167.75 \\
\hline 5 & 9.45 & 9.80 & 15.90 & 23.30 & 42.78 & 47.47 & 56.25 & 74.94 & 45.56 & 60.59 & 128.40 & 167.75 \\
\hline 6 & 9.45 & 9.80 & 15.90 & 23.30 & 42.77 & 47.47 & 56.23 & 74.94 & 45.56 & 60.59 & 128.40 & 167.75 \\
\hline 8 & 9.45 & 9.80 & 15.90 & 23.30 & 42.77 & 47.47 & 56.21 & 74.94 & 45.56 & 60.59 & 128.40 & 167.75 \\
\hline 10 & 9.45 & 9.80 & 15.90 & 23.30 & 42.77 & 47.47 & 56.21 & 74.94 & 45.56 & 60.59 & 128.40 & 167.75 \\
\hline
\end{tabular}

Table 1 and all the following examples, Jacobian orthogonal polynomials are uniformly expanded into eight terms. The results of the convergence study of weighted parameters are shown in Table 3. From Table 3, it can be found that when the weighted parameter is small, the result is unstable. With the increase of weighted parameters, the calculated results converge rapidly. When the weighted parameter is more than $10^{20}$, the results diverge. Therefore, it is indicated that when the weighted parameters are set between $10^{14}$ and $10^{18}$, stable converged results can be obtained. In all subsequent examples, the weighted parameters are set as $10^{14}$.

Based on the study of convergence, the correctness of the present method is further studied. Table 4 shows the numerical comparison of Sandwich FGP doubly curved structure with various boundary conditions. The geometric parameters are as follows: elliptical Shell: $a_{1}=1 \mathrm{~m}, b_{1}=2 \mathrm{~m}$, $R_{s}=1 m, h=0.1 m, \varphi_{0}=\pi / 6, \varphi_{0}=5 \pi / 6$; hyperbolic shell: $R_{1}=2 m, R_{s}=0 m, c=1 m, D=4 m, h=0.1 m$. The dimensionless frequencies are as follows: $\Omega=\omega R_{s} / h \sqrt{\rho_{1} / E_{1}}(\mathrm{el}-$ liptical Shell) and $\Omega=\omega D^{2} \sqrt{\rho_{1} / E_{1}}$ (hyperbolic shell). Sandwich material type is $1-2$, and the thickness coefficient of Sandwich structure is defined as 0-1-0. Table 4 shows that the results obtained in this paper are very close to those obtained by Zhao et al. [35] using Ritz method. Table 5 gives the comparative data of Sandwich FGP doubly curved elliptical shell, which are obtained from finite element method (FEM) through ABAQUS. The boundary condition is defined as F-C. Geometric parameters are defined as follows: $a_{1}=1 m, b_{1}=2 m, R_{s}=0 m, h=0.1 m, \varphi_{0}=0, \varphi_{0}=\pi / 2$. The Sandwich material type and thickness coefficient are consistent with those in Table 1 . Table 5 shows that the present method is appropriate for calculating free vibration features of Sandwich FGP doubly curved shells.
Next, the correctness of this method for forced response prediction is further verified. Due to the lack of relevant literature results, the finite element software ABAQUS is used. The geometric dimensions and material parameters are identical with those in Table 5. Figure 4 shows three loads: point load, line load, and surface load. In the steady-state response analysis, the load form is point load, and the position of action and observation point are $A(\varphi, \theta)=([0,0],[0,0])$ and $O(\varphi, \theta)=([\pi / 4, \pi / 4],[0,0])$, respectively. It should be noted that the first item in parentheses represents generatrix direction $\varphi$ and the second one represents circumferential direction $\theta$. The point load is as follows: $f_{w}=\bar{f}_{w} \delta\left(\varphi-\varphi_{A}\right)\left(\theta-\theta_{A}\right)$, where $\bar{f}_{w}=-1 N$, and $\delta$ is the Dirac delta function. Frequency ranges from $500 \mathrm{~Hz}$ to $1200 \mathrm{~Hz}$, with a total of 501 sweeps. Figure 5 shows four specific forms of impact load function: (a) rectangular pulse signal; (b) triangular pulse signal; (c) half-sine pulse signal; and (d) exponential pulse signal, respectively [36]. In transient response analysis, the shock load function is chosen as rectangular wave. The loading time is $10 \mathrm{~ms}$, and the interpolation point is 501. The loading position is consistent with that of the steady-state response, and the coordinates of observation points are defined as $(\pi / 3,0)$. Figure 6 shows the comparison of steady-state response and transient response, respectively. It is obvious from Figure 6 that the proposed method has excellent accuracy for the prediction of forced response of Sandwich FGP doubly curved structure.

\section{Numerical Results and Discussion}

The third part has made a detailed comparative study on the correctness of the theoretical modeling, which verifies that the proposed model has excellent convergence speed and 
TABLE 3: Convergence analysis of weighted parameters for Sandwich FGP doubly curved structure.

\begin{tabular}{|c|c|c|c|c|c|c|c|c|c|c|c|c|}
\hline \multirow{2}{*}{$\Lambda$} & \multicolumn{4}{|c|}{ Paraboloidal shell } & \multicolumn{4}{|c|}{ Hyperbolic shell } & \multicolumn{4}{|c|}{ Elliptical shell } \\
\hline & 1 & 2 & 3 & 4 & 1 & 2 & 3 & 4 & 1 & 2 & 3 & 4 \\
\hline $10^{8}$ & 3.84 & 5.97 & 8.46 & 14.23 & 9.03 & 14.49 & 22.34 & 23.16 & 17.95 & 38.69 & 39.41 & 39.85 \\
\hline $10^{10}$ & 9.01 & 9.27 & 15.86 & 21.59 & 55.41 & 60.98 & 69.31 & 82.13 & 45.20 & 55.59 & 126.38 & 154.68 \\
\hline $10^{12}$ & 9.45 & 9.79 & 15.90 & 23.28 & 42.86 & 47.59 & 56.33 & 75.07 & 45.56 & 60.53 & 128.38 & 167.61 \\
\hline $10^{14}$ & 9.45 & 9.80 & 15.90 & 23.30 & 42.79 & 47.47 & 56.30 & 74.93 & 45.56 & 60.59 & 128.40 & 167.75 \\
\hline $10^{16}$ & 9.45 & 9.80 & 15.90 & 23.30 & 42.79 & 47.47 & 56.30 & 74.93 & 45.56 & 60.59 & 128.40 & 167.75 \\
\hline $10^{18}$ & 9.45 & 9.80 & 15.90 & 23.30 & 42.79 & 47.47 & 56.30 & 74.93 & 45.56 & 60.59 & 128.39 & 167.75 \\
\hline $10^{20}$ & 9.45 & 9.83 & 15.88 & 23.30 & 42.71 & 47.46 & 56.29 & 74.93 & 45.68 & 60.55 & 128.48 & 167.76 \\
\hline $10^{22}$ & 9.83 & 21.09 & 27.28 & 35.32 & 57.04 & 64.68 & 66.65 & 77.95 & 46.32 & 71.05 & 170.45 & 174.49 \\
\hline $10^{24}$ & 131.67 & 134.72 & 160.47 & 169.09 & 140.82 & 181.57 & 200.96 & 210.62 & 369.36 & 445.81 & 481.35 & 566.60 \\
\hline
\end{tabular}

TABLE 4: Numerical comparison of frequencies for Sandwich FGP doubly curved structure with various boundary conditions.

\begin{tabular}{|c|c|c|c|c|c|c|c|c|c|}
\hline \multirow{3}{*}{ Type } & \multirow{3}{*}{ Mode } & \multicolumn{4}{|c|}{ Elliptical shell } & \multicolumn{4}{|c|}{ Hyperbolic shell } \\
\hline & & \multicolumn{2}{|r|}{$\mathrm{C}-\mathrm{C}$} & \multicolumn{2}{|r|}{$\mathrm{C}-\mathrm{F}$} & \multicolumn{2}{|r|}{$\mathrm{C}-\mathrm{C}$} & \multicolumn{2}{|r|}{ C-F } \\
\hline & & Present & Reference [35] & Present & Reference [35] & Present & Reference [35] & Present & Reference [35] \\
\hline \multirow{4}{*}{ Type1-1 } & 1 & 3.01 & 3.009 & 0.574 & 0.574 & 2.687 & 2.68 & 1.839 & 1.838 \\
\hline & 2 & 3.012 & 3.011 & 0.738 & 0.738 & 3.025 & 3.013 & 2.218 & 2.217 \\
\hline & 3 & 3.14 & 3.14 & 1.612 & 1.612 & 4.182 & 4.151 & 2.633 & 2.627 \\
\hline & 4 & 3.147 & 3.146 & 2.028 & 2.029 & 4.404 & 4.386 & 2.984 & 2.974 \\
\hline \multirow{4}{*}{ Type1-2 } & 1 & 3.003 & 3.004 & 0.562 & 0.563 & 2.671 & 2.665 & 1.814 & 1.814 \\
\hline & 2 & 3.005 & 3.006 & 0.735 & 0.735 & 2.969 & 2.959 & 2.217 & 2.217 \\
\hline & 3 & 3.129 & 3.129 & 1.581 & 1.582 & 4.087 & 4.058 & 2.613 & 2.607 \\
\hline & 4 & 3.138 & 3.139 & 2.028 & 2.03 & 4.333 & 4.31 & 2.925 & 2.916 \\
\hline
\end{tabular}

TABLE 5: Numerical comparison of frequencies for Sandwich FGP elliptical shell accompanied with F-C boundary condition.

\begin{tabular}{lcccccccccc}
\hline Mode & 1 & 2 & 3 & 4 & 5 & 6 & 7 & 8 & 9 & 10 \\
\hline Present & 272.39 & 420.47 & 466.94 & 550.27 & 591.89 & 595.34 & 701.03 & 712.70 & 757.62 & 789.95 \\
FEM & 273.15 & 420.2 & 466.42 & 553.54 & 592.22 & 595.2 & 703.33 & 712.35 & 757.27 & 790.86 \\
\hline
\end{tabular}
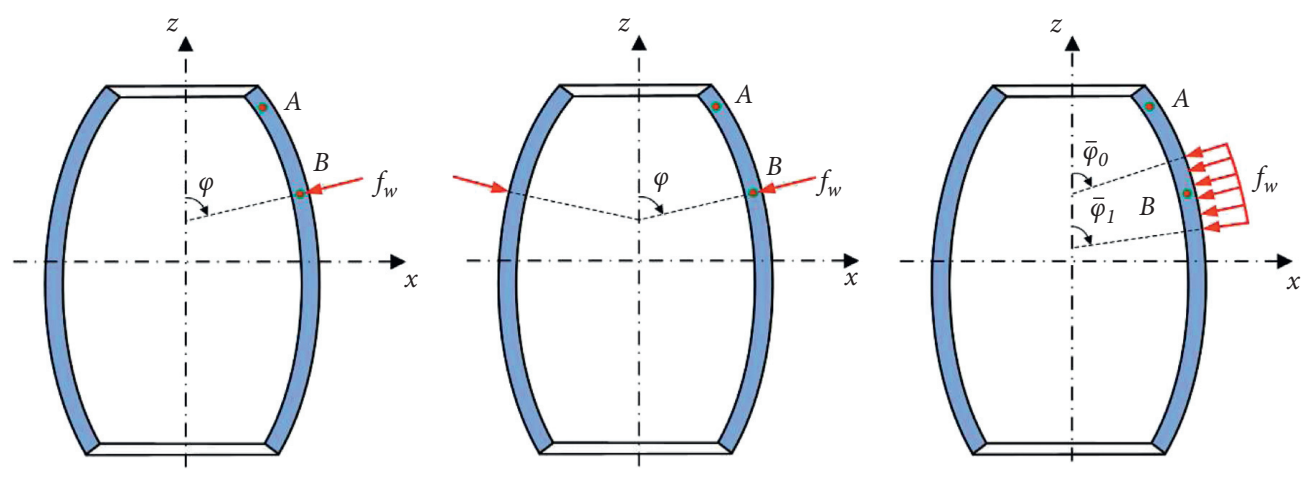



(a)



(b)

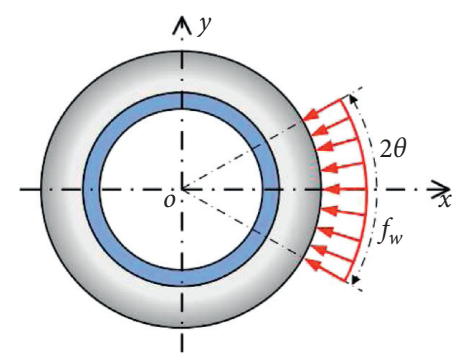

(c)

FIgURE 4: Schematic diagram of three categories of loads. (a) Point force. (b) Line force. (c) Surface force. 




(a)



(b)

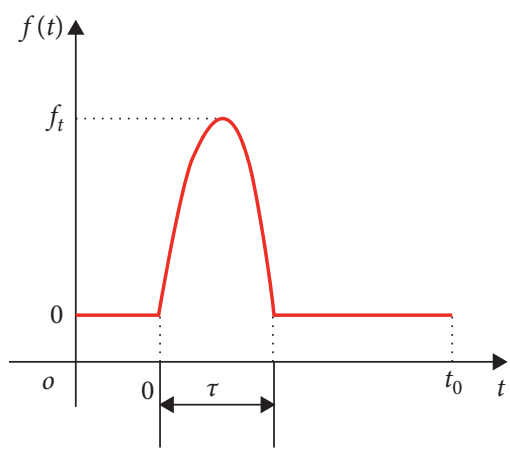

(c)

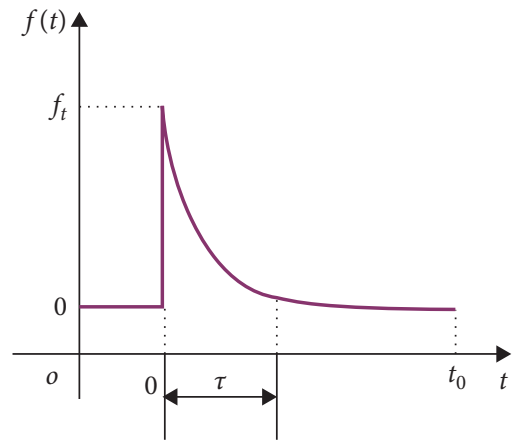

(d)

Figure 5: The diagram of load signal. (a) Rectangular pulse signal. (b) Triangular pulse signal. (c) Half-sine pulse signal. (d) Exponential pulse signal.



(a)

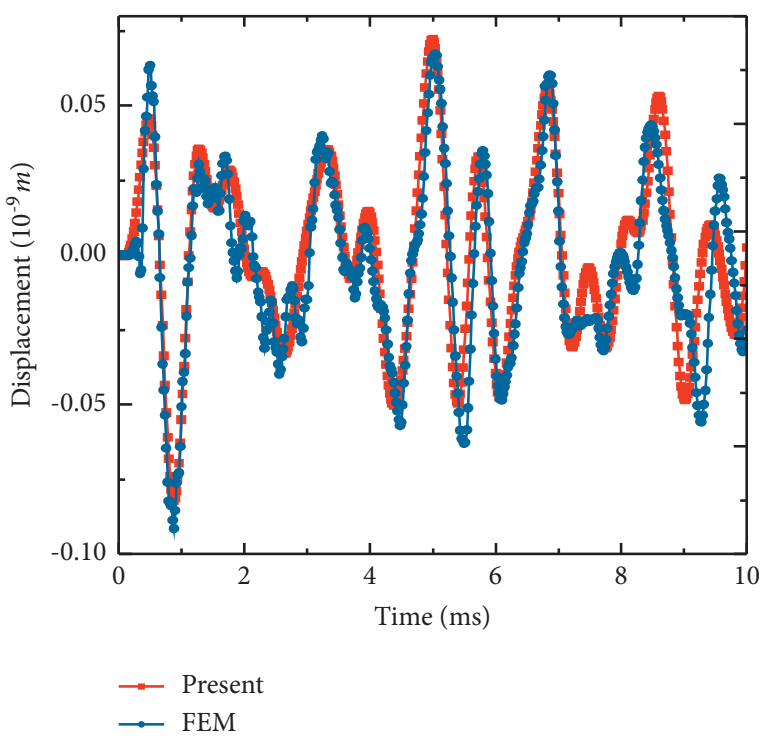

(b)

FIGURE 6: Verification of the correctness of responses: (a) steady-state response; (b) transient response.

computational accuracy. In this part, numerical discussions will be further carried out.

Tables 6 and 7 show the first four frequencies of four Sandwich FGP materials, respectively. The geometric dimensions are as follows: paraboloidal shell: Case 1: $R_{0}=2 \mathrm{~m}$,
$R_{1}=4 \mathrm{~m}, R_{s}=1 \mathrm{~m}, k=1, h=0.1 \mathrm{~m}$; Case $2: R_{0}=2 \mathrm{~m}, R_{1}=4 \mathrm{~m}$, $R_{s}=1 \mathrm{~m}, k=2, h=0.1 \mathrm{~m}$; Case $3: R_{0}=2 \mathrm{~m}, R_{1}=4 \mathrm{~m}, R_{s}=1 \mathrm{~m}$, $k=3, h=0.1 \mathrm{~m}$; hyperbolic shell: Case $1: R_{1}=2 \mathrm{~m}, R_{s}=1 \mathrm{~m}$, $c=1 \mathrm{~m}, D=4 \mathrm{~m}, h=0.1 \mathrm{~m}$; Case $2: R_{1}=2 \mathrm{~m}, R_{s}=1 \mathrm{~m}, c=2 \mathrm{~m}$, $D=4 m, h=0.1 m$; Case $3: R_{1}=2 m, R_{s}=1 m, c=3 m, D=4 m$, 
TABLE 6: First four frequencies for Type 1-1 Sandwich FGP doubly curved structure with diverse boundary conditions.

\begin{tabular}{|c|c|c|c|c|c|c|c|c|c|c|}
\hline \multirow{2}{*}{ Type } & \multirow{2}{*}{ Mode } & \multicolumn{3}{|c|}{ Parabolic shell } & \multicolumn{3}{|c|}{ Hyperbolic shell } & \multicolumn{3}{|c|}{ Elliptical shell } \\
\hline & & $\mathrm{CC}$ & SS & $\mathrm{CF}$ & $\mathrm{CC}$ & SS & $\mathrm{CF}$ & $\mathrm{CC}$ & SS & $\mathrm{CF}$ \\
\hline \multirow{4}{*}{ Case 1} & 1 & 66.05 & 63.40 & 15.27 & 107.90 & 105.24 & 43.61 & 361.55 & 276.58 & 71.18 \\
\hline & 2 & 67.39 & 64.72 & 18.55 & 112.79 & 109.18 & 47.32 & 389.58 & 315.40 & 130.70 \\
\hline & 3 & 72.28 & 69.69 & 23.88 & 135.21 & 132.53 & 58.47 & 461.71 & 407.96 & 146.15 \\
\hline & 4 & 77.90 & 75.54 & 27.64 & 139.24 & 133.68 & 74.45 & 551.69 & 515.53 & 220.14 \\
\hline \multirow{4}{*}{ Case 2} & 1 & 118.03 & 109.84 & 23.74 & 100.64 & 98.62 & 41.48 & 247.30 & 219.58 & 47.23 \\
\hline & 2 & 120.89 & 112.10 & 25.83 & 110.98 & 107.24 & 42.22 & 247.48 & 247.10 & 60.64 \\
\hline & 3 & 121.30 & 114.13 & 31.06 & 120.62 & 118.73 & 58.03 & 258.01 & 247.46 & 132.53 \\
\hline & 4 & 131.65 & 125.87 & 41.13 & 139.31 & 133.31 & 64.42 & 258.59 & 257.41 & 166.64 \\
\hline \multirow{4}{*}{ Case 3} & 1 & 162.09 & 143.75 & 29.26 & 97.21 & 95.55 & 39.66 & 135.46 & 135.32 & 35.27 \\
\hline & 2 & 163.01 & 144.21 & 31.77 & 108.38 & 105.47 & 39.99 & 137.59 & 137.31 & 43.47 \\
\hline & 3 & 165.72 & 148.30 & 36.00 & 109.28 & 107.08 & 57.33 & 153.59 & 153.53 & 111.17 \\
\hline & 4 & 168.36 & 149.76 & 45.25 & 115.59 & 112.43 & 57.87 & 157.70 & 157.48 & 120.46 \\
\hline
\end{tabular}

TABle 7: First four frequencies for Type 1-2 Sandwich FGP doubly curved structure with diverse boundary conditions.

\begin{tabular}{|c|c|c|c|c|c|c|c|c|c|c|}
\hline \multirow{2}{*}{ Type } & \multirow{2}{*}{ Mode } & \multicolumn{3}{|c|}{ Parabolic shell } & \multicolumn{3}{|c|}{ Hyperbolic shell } & \multicolumn{3}{|c|}{ Elliptical shell } \\
\hline & & CC & SS & CF & CC & SS & CF & $\mathrm{CC}$ & SS & $\mathrm{CF}$ \\
\hline \multirow{4}{*}{ Case 1} & 1 & 64.52 & 61.79 & 14.92 & 105.94 & 102.88 & 42.55 & 352.10 & 267.85 & 69.38 \\
\hline & 2 & 66.22 & 63.48 & 17.88 & 109.70 & 105.77 & 46.70 & 379.98 & 307.29 & 126.54 \\
\hline & 3 & 70.19 & 67.54 & 23.57 & 133.47 & 128.71 & 56.51 & 451.42 & 400.48 & 143.67 \\
\hline & 4 & 76.83 & 74.43 & 26.56 & 134.57 & 130.34 & 73.61 & 539.98 & 507.61 & 216.17 \\
\hline \multirow{4}{*}{ Case 2} & 1 & 115.63 & 107.99 & 22.98 & 98.74 & 96.44 & 40.40 & 243.99 & 213.51 & 45.66 \\
\hline & 2 & 118.02 & 109.71 & 25.32 & 107.84 & 103.78 & 41.64 & 244.17 & 243.90 & 59.70 \\
\hline & 3 & 119.20 & 112.62 & 29.90 & 119.08 & 116.81 & 56.05 & 254.14 & 243.95 & 128.40 \\
\hline & 4 & 129.66 & 124.50 & 40.56 & 134.59 & 128.31 & 63.70 & 254.95 & 254.14 & 164.83 \\
\hline \multirow{4}{*}{ Case 3} & 1 & 158.57 & 142.09 & 28.32 & 95.34 & 93.42 & 38.88 & 133.21 & 133.08 & 34.73 \\
\hline & 2 & 159.15 & 143.02 & 31.05 & 106.03 & 101.91 & 39.10 & 135.84 & 135.60 & 41.98 \\
\hline & 3 & 162.41 & 145.99 & 34.67 & 107.00 & 105.37 & 55.87 & 149.92 & 149.87 & 109.98 \\
\hline & 4 & 164.02 & 148.88 & 44.51 & 113.32 & 109.84 & 56.69 & 155.88 & 155.69 & 117.50 \\
\hline
\end{tabular}

$h=0.1 m$; Elliptical shell: Case 1: $a_{1}=1 m, b_{1}=1 m, R_{s}=1 m$, $h=0.1 m, \varphi_{0}=\pi / 6, \varphi_{0}=5 \pi / 6 ;$ Case $2: a_{1}=1 m, b_{1}=2 m$, $R_{s}=1 \mathrm{~m}, h=0.1 \mathrm{~m}, \varphi_{0}=\pi / 6, \varphi_{0}=5 \pi / 6$; Case 3: $a_{1}=1 \mathrm{~m}$, $b_{1}=3 m, R_{s}=1 m, h=0.1 m, \varphi_{0}=\pi / 6, \varphi_{0}=5 \pi / 6$. For parabolic shells, the frequency parameters of shells increase with the increase of characteristic coefficient $k$. For hyperbolic shells, when the structure coefficient $c$ increases, the frequency parameters of the shells decrease. For elliptical shells, when the structural coefficient $b$ increases, the frequency parameters decrease accordingly. The above results indicate that the change of parameters directly affects the stiffness matrix of the Sandwich FGP doubly curved structure. Besides, the frequency parameters of Type 1--1 Sandwich FGP materials are the largest, while those of Type 1-2 Sandwich materials are the smallest. For the study of free vibration, modal modes can provide more information. Therefore, modal modes under some elastic boundary conditions are given in Figures 7, 8, 9.

Next, the parameters of the forced response are examined. The geometric parameters are consistent with those of Case 1 in Tables 6, 7, 8, and 9. In the steady-state response, the load type is linear. The boundary is defined as $\mathrm{C}-\mathrm{C}$, and the Sandwich FGP material is selected as Types 1-2. For different doubly curved structures, the location of load action is defined as follows: paraboloidal shell:
$A(\varphi, \theta)=([\pi / 3,2 \pi / 3],[0, \pi]) ; \quad$ hyperbolic shell: $A(\varphi, \theta)=([\pi / 2, \pi / 2],[0, \pi]) ; \quad$ and elliptical shell: $A(\varphi, \theta)=([\pi / 2, \pi / 2],[0, \pi])$. The locations of observation point are defined as follows: paraboloidal shell: $O(\varphi, \theta)=([\pi / 3, \pi / 3],[0,0]) ; \quad$ hyperbolic shell: $O(\varphi, \theta)=([\pi / 4, \pi / 4],[0,0]) ; \quad$ and elliptical shell: $O(\varphi, \theta)=([\pi / 4, \pi / 4],[0,0])$. In order to simplify the transient study, the shock load function is chosen as rectangular wave. The position of the impact load is consistent with that of the steady-state response. Similarly, observation points are defined as follows: paraboloidal shell: $O(\varphi, \theta)=([3 \pi / 10,3 \pi / 10],[0,0]) ; \quad$ hyperbolic shell: $O(\varphi, \theta)=([\pi / 2, \pi / 2],[0,0]) ;$ and elliptical shell: $O(\varphi, \theta)=([\pi / 4, \pi / 4],[0,0])$.

Figures 10 and 11, respectively, study the steady and transient responses of the structure under different elastic boundary conditions. It can be found that the boundary condition parameters have a significant effect on the forced response of the Sandwich FGP doubly curved structure. Figure 12 and 13, respectively, present the steady and transient responses of the Sandwich FGP doubly curved structure under different porous coefficient. For steady-state response analysis, with the same frequency range, the number of resonance peaks decreases with the increase of pore coefficient. For transient 


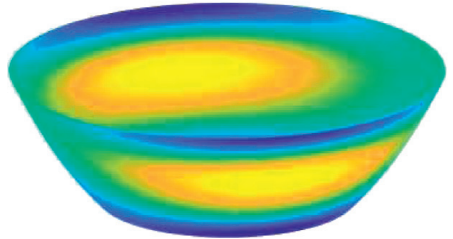

Mode $=(1,1)$

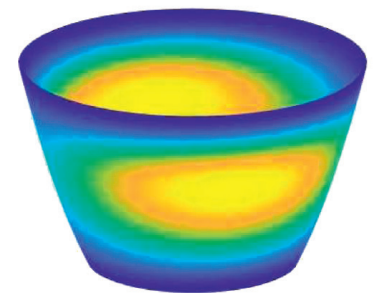

Mode $=(1,1)$

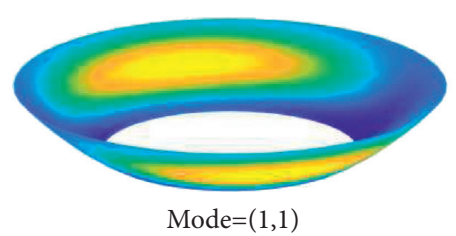

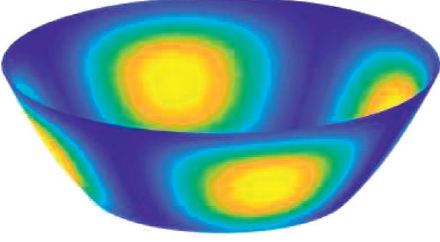

Mode $=(1,2)$

(a)

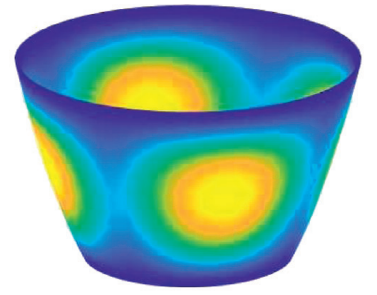

Mode $=(1,2)$

(b)

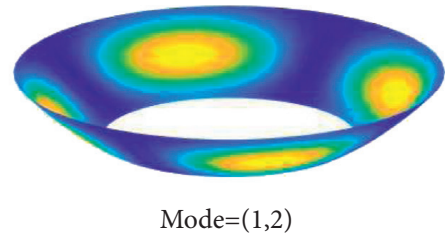

(c)

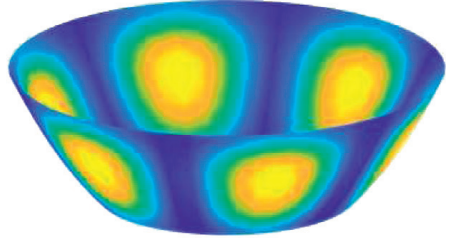

Mode $=(1,3)$



Mode $=(1,3)$

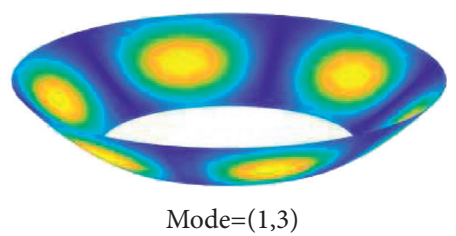

FIGURE 7: Several typical mode shapes for Sandwich FGP paraboloidal shell. (a) Paraboloidal shell $(k=1)$ with E1-E1 boundary conditions. (b) Paraboloidal shell $(k=2)$ with E2-E2 boundary conditions. (c) Paraboloidal shell $(k=3)$ with E3-E3 boundary conditions.

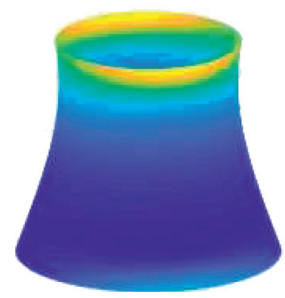

Mode $=(1,1)$

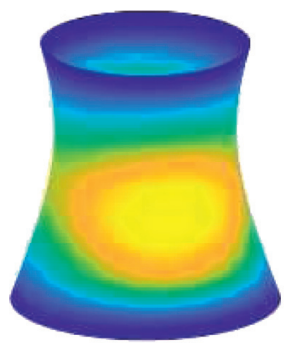

Mode $=(1,1)$



Mode $=(1,2)$

(a)

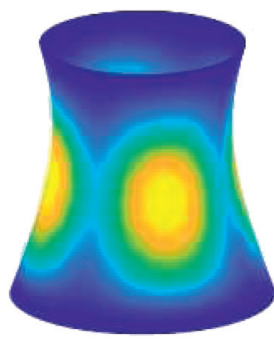

Mode $=(1,2)$

(b)
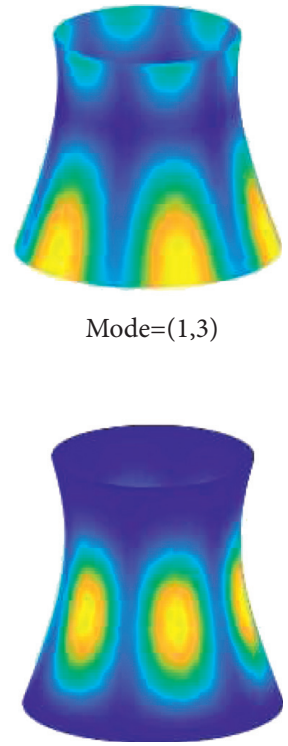

Mode $=(1,3)$

Figure 8: Continued. 


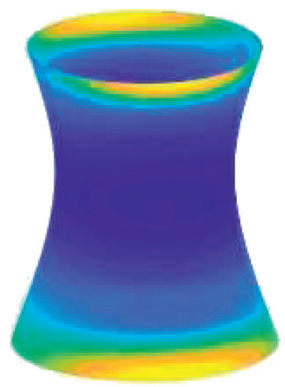

Mode $=(1,1)$

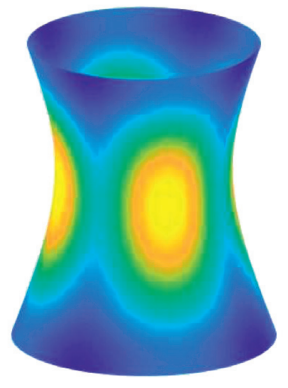

Mode $=(1,2)$

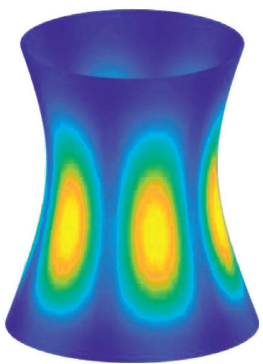

Mode $=(1,3)$

(c)

FIgURE 8: Several typical mode shapes for Sandwich FGP hyperbolic shell. (a) Hyperbolic shell $(c=1)$ with E1-E1 boundary conditions. (b) Hyperbolic shell $(c=2)$ with E2-E2 boundary conditions. (c) Hyperbolic shell $(c=3)$ with E3-E3 boundary conditions.

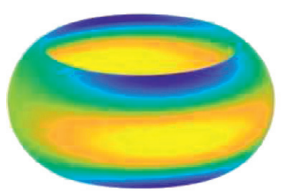

Mode $=(1,1)$

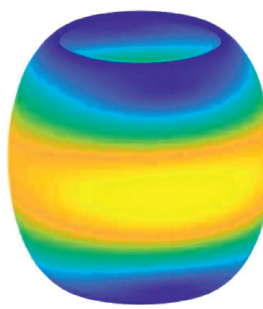

Mode $=(1,1)$

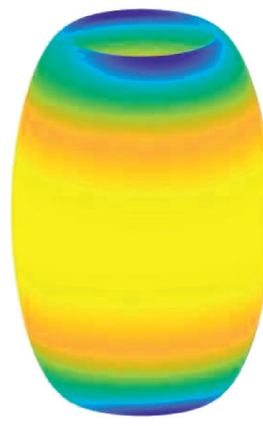

Mode $=(1,1)$

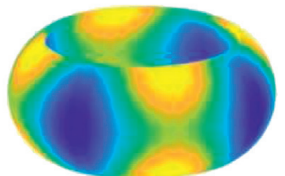

Mode $=(1,2)$

(a)

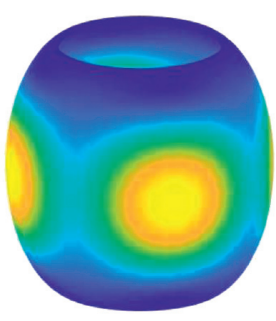

Mode $=(1,2)$

(b)

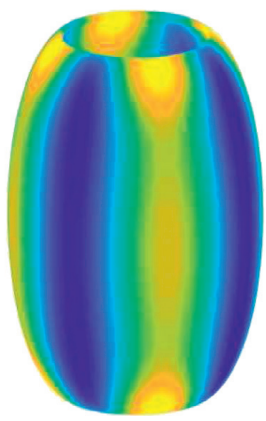

Mode $=(1,2)$



Mode $=(1,3)$



Mode $=(1,3)$

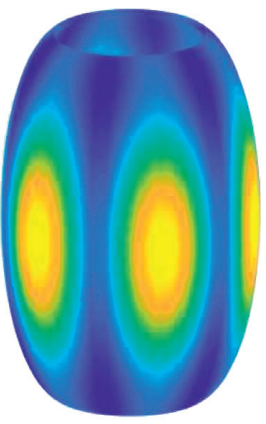

Mode $=(1,3)$

(c)

FIGURE 9: Several typical mode shapes for Sandwich FGP elliptical shell. (a) Elliptical shell $(b=1)$ with E1-E1 boundary conditions. (b) Elliptical shell $(b=2)$ with E1-E1 boundary conditions. (c) Elliptical shell $(b=3)$ with E1-E1 boundary conditions.

TABLE 8: First four frequencies for Type 2-1 Sandwich FGP doubly curved structure with diverse boundary conditions.

\begin{tabular}{|c|c|c|c|c|c|c|c|c|c|c|}
\hline \multirow{2}{*}{ Type } & \multirow{2}{*}{ Mode } & \multicolumn{3}{|c|}{ Parabolic shell } & \multicolumn{3}{|c|}{ Hyperbolic shell } & \multicolumn{3}{|c|}{ Elliptical shell } \\
\hline & & $\mathrm{CC}$ & SS & $\mathrm{CF}$ & $\mathrm{CC}$ & SS & $\mathrm{CF}$ & $\mathrm{CC}$ & SS & $\mathrm{CF}$ \\
\hline \multirow{4}{*}{ Case 1} & 1 & 64.94 & 62.44 & 15.01 & 107.20 & 104.70 & 42.79 & 354.72 & 273.62 & 69.75 \\
\hline & 2 & 67.03 & 64.52 & 17.74 & 110.00 & 106.64 & 47.47 & 383.30 & 313.01 & 126.40 \\
\hline & 3 & 70.23 & 67.79 & 23.95 & 134.10 & 128.93 & 56.30 & 456.33 & 406.35 & 145.66 \\
\hline & 4 & 78.04 & 75.83 & 26.29 & 135.71 & 133.17 & 74.93 & 546.42 & 513.89 & 218.24 \\
\hline
\end{tabular}


TABle 8: Continued.

\begin{tabular}{|c|c|c|c|c|c|c|c|c|c|c|}
\hline \multirow{2}{*}{ Type } & \multirow{2}{*}{ Mode } & \multicolumn{3}{|c|}{ Parabolic shell } & \multicolumn{3}{|c|}{ Hyperbolic shell } & \multicolumn{3}{|c|}{ Elliptical shell } \\
\hline & & $\mathrm{CC}$ & SS & $\mathrm{CF}$ & $\mathrm{CC}$ & SS & $\mathrm{CF}$ & $\mathrm{CC}$ & SS & $\mathrm{CF}$ \\
\hline \multirow{4}{*}{ Case 2} & 1 & 116.71 & 109.14 & 22.92 & 99.86 & 97.95 & 40.55 & 248.11 & 219.33 & 45.56 \\
\hline & 2 & 118.70 & 110.50 & 25.56 & 108.03 & 104.53 & 42.31 & 248.57 & 248.07 & 60.59 \\
\hline & 3 & 120.66 & 114.08 & 29.62 & 121.11 & 119.30 & 55.79 & 257.72 & 248.33 & 128.40 \\
\hline & 4 & 131.52 & 126.25 & 40.85 & 134.06 & 128.50 & 64.85 & 259.37 & 257.72 & 167.75 \\
\hline \multirow{4}{*}{ Case 3} & 1 & 159.78 & 142.49 & 28.23 & 96.39 & 94.80 & 38.96 & 135.12 & 134.99 & 35.26 \\
\hline & 2 & 160.05 & 143.63 & 31.25 & 106.05 & 102.53 & 39.72 & 138.25 & 138.01 & 41.87 \\
\hline & 3 & 163.93 & 146.16 & 34.37 & 108.83 & 107.57 & 55.60 & 151.02 & 150.98 & 111.92 \\
\hline & 4 & 164.60 & 149.66 & 45.07 & 114.48 & 111.54 & 57.71 & 158.70 & 158.51 & 118.26 \\
\hline
\end{tabular}

TABle 9: First four frequencies for Type 2-2 Sandwich FGP doubly curved structure with diverse boundary conditions.

\begin{tabular}{|c|c|c|c|c|c|c|c|c|c|c|}
\hline \multirow{2}{*}{ Type } & \multirow{2}{*}{ Mode } & \multicolumn{3}{|c|}{ Parabolic shell } & \multicolumn{3}{|c|}{ Hyperbolic shell } & \multicolumn{3}{|c|}{ Elliptical shell } \\
\hline & & $\mathrm{CC}$ & SS & $\mathrm{CF}$ & $\mathrm{CC}$ & SS & $\mathrm{CF}$ & $\mathrm{CC}$ & SS & $\mathrm{CF}$ \\
\hline \multirow{4}{*}{ Case 1} & 1 & 65.07 & 62.53 & 15.04 & 107.44 & 104.85 & 42.87 & 355.24 & 273.61 & 69.86 \\
\hline & 2 & 67.19 & 64.64 & 17.76 & 110.18 & 106.73 & 47.59 & 383.91 & 313.29 & 126.55 \\
\hline & 3 & 70.34 & 67.86 & 24.01 & 134.25 & 128.98 & 56.36 & 457.13 & 407.18 & 145.98 \\
\hline & 4 & 78.23 & 76.00 & 26.31 & 136.07 & 133.42 & 75.14 & 547.42 & 515.20 & 218.49 \\
\hline \multirow{4}{*}{ Case 2} & 1 & 116.95 & 109.41 & 22.95 & 100.08 & 98.10 & 40.62 & 248.72 & 219.35 & 45.61 \\
\hline & 2 & 118.92 & 110.73 & 25.62 & 108.20 & 104.61 & 42.41 & 249.20 & 248.68 & 60.73 \\
\hline & 3 & 120.94 & 114.40 & 29.65 & 121.42 & 119.53 & 55.85 & 258.30 & 248.95 & 128.55 \\
\hline & 4 & 131.84 & 126.63 & 40.88 & 134.21 & 128.54 & 65.03 & 259.95 & 258.30 & 168.22 \\
\hline \multirow{4}{*}{ Case 3} & 1 & 160.10 & 143.00 & 28.26 & 96.59 & 94.95 & 39.02 & 135.43 & 135.30 & 35.34 \\
\hline & 2 & 160.35 & 144.19 & 31.32 & 106.20 & 102.58 & 39.82 & 138.61 & 138.37 & 41.91 \\
\hline & 3 & 164.28 & 146.63 & 34.40 & 109.11 & 107.78 & 55.66 & 151.29 & 151.24 & 112.23 \\
\hline & 4 & 164.89 & 150.28 & 45.18 & 114.72 & 111.70 & 57.86 & 159.12 & 158.94 & 118.45 \\
\hline
\end{tabular}

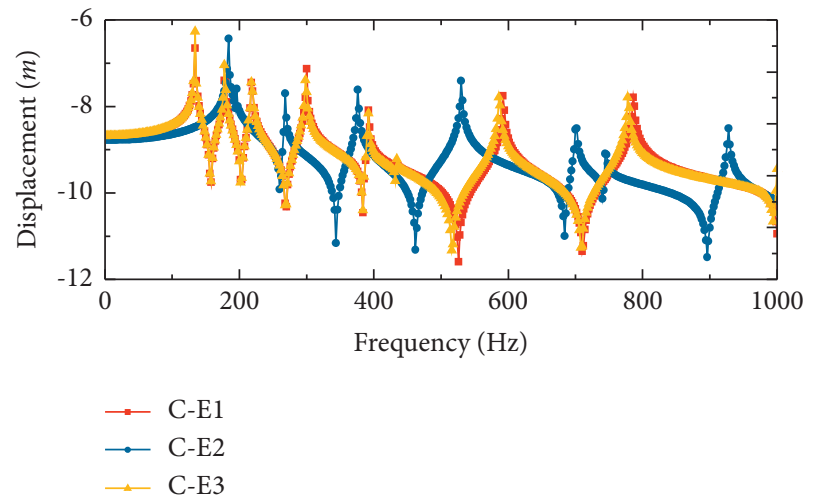

(a)

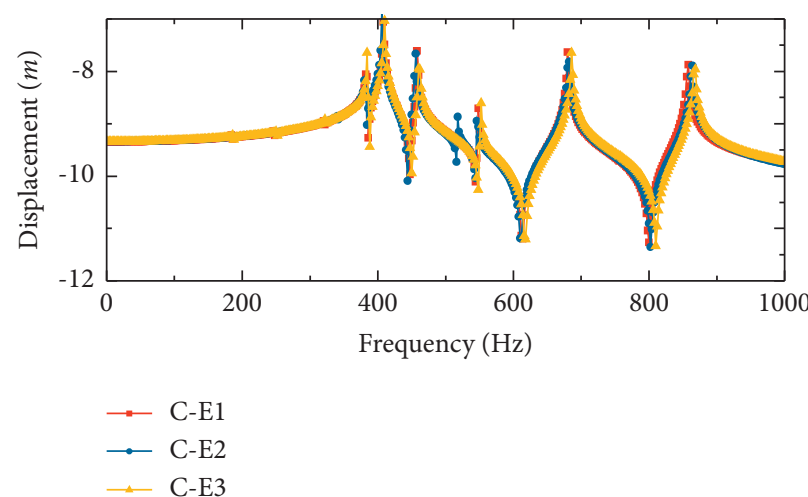

(b)

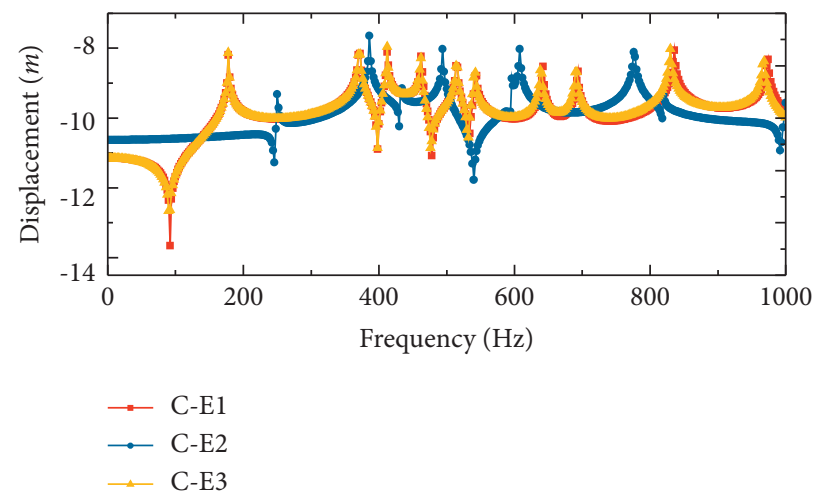

(c)

FIGURE 10: Study on the influence of elastic boundary conditions on the steady-state response of Sandwich FGP doubly curved structure: (a) Sandwich FGP paraboloidal shell; (b) Sandwich FGP hyperbolic shell; (c) Sandwich FGP elliptical shell. 

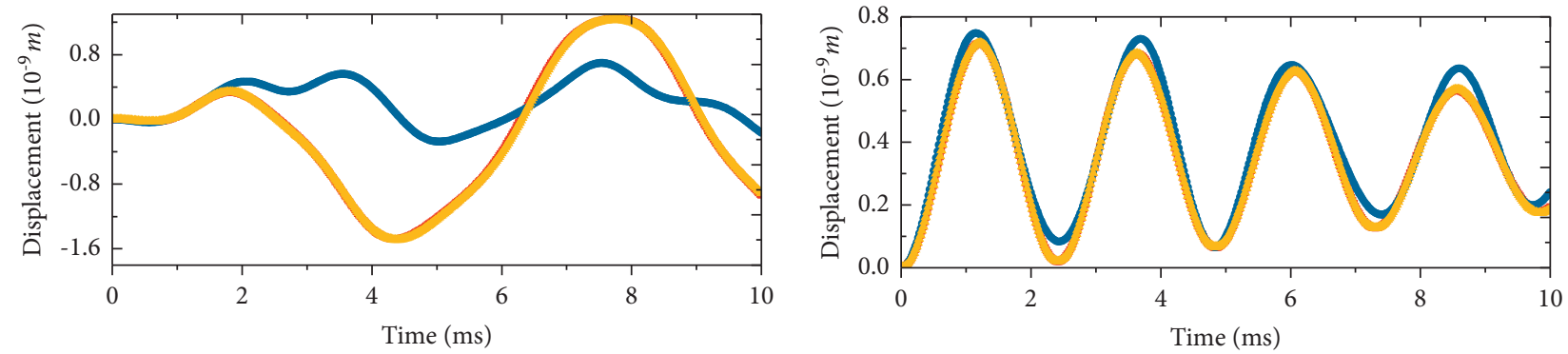

$\rightarrow$ C-E1
$\rightarrow$ C-E2
$\rightarrow$ C-E3

$\because \mathrm{C}-\mathrm{E} 1$

$\rightarrow \mathrm{C}-\mathrm{E} 2$

$\rightarrow$ C-E3

(a)

(b)



(c)

FIGURE 11: Study on the influence of elastic boundary conditions on the transient response of Sandwich FGP doubly curved structure: (a) Sandwich FGP paraboloidal shell; (b) Sandwich FGP hyperbolic shell; (c) Sandwich FGP elliptical shell.

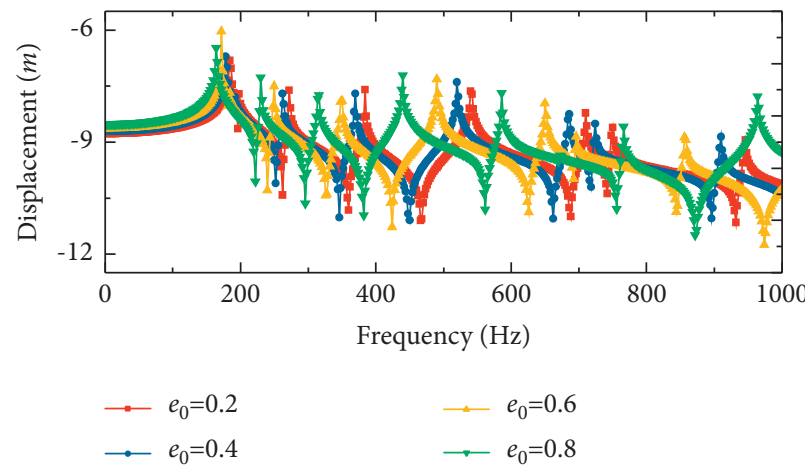

(a)



(b)

FIgURE 12: Continued. 


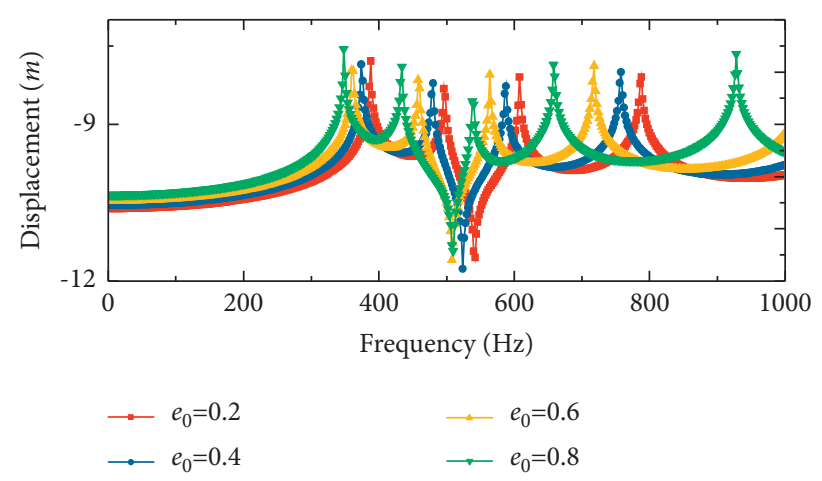

(c)

FIGURE 12: Study on the effect of porous coefficient on the steady-state response of Sandwich FGP doubly curved structure: (a) Sandwich FGP paraboloidal shell; (b) Sandwich FGP hyperbolic shell; (c) Sandwich FGP elliptical shell.

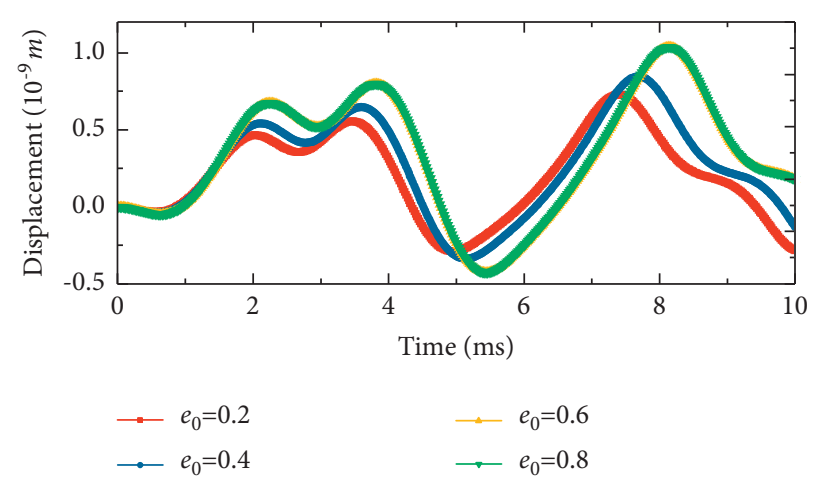

(a)

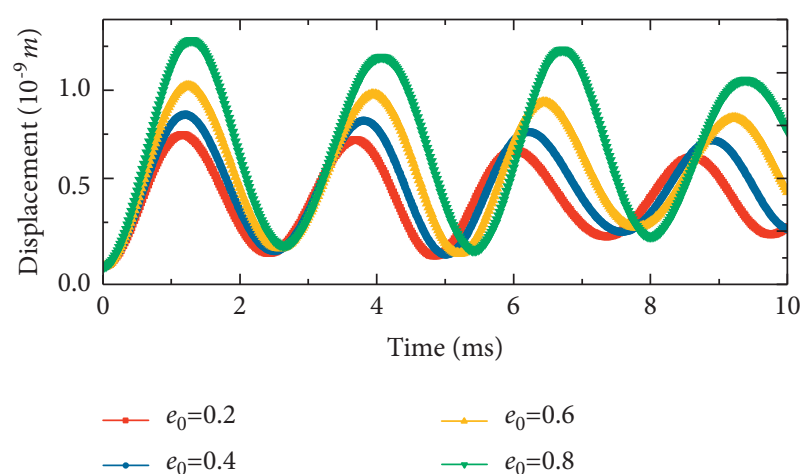

(b)

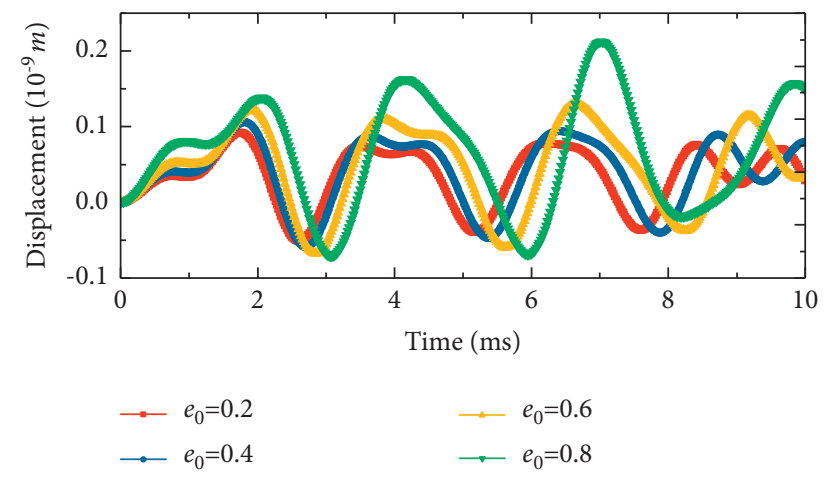

(c)

FIGURE 13: Study on the effect of porous coefficient on the transient response of Sandwich FGP doubly curved structure: (a) Sandwich FGP paraboloidal shell; (b) Sandwich FGP hyperbolic shell; (c) Sandwich FGP elliptical shell.

response analysis, the increase of porous coefficient results in the increase of response amplitude to a certain extent. The main reason for this is that the increase of porous coefficient can reduce the quality of the structure. Figures 14 and 15 show the effect of the thickness of FGP materials on the forced response of Sandwich FGP doubly curved structure. From these two graphs, it is observed that the thickness coefficient of FGP layer is not very sensitive to the forced response of the Sandwich FGP doubly curved structure. For the steady-state response, the 


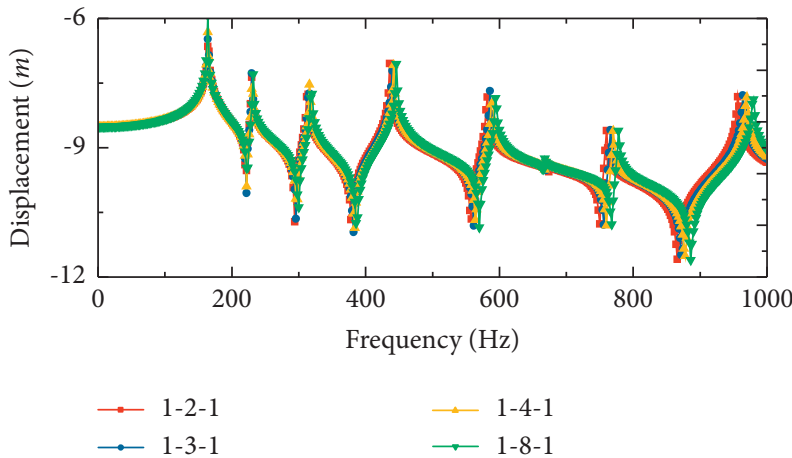

(a)

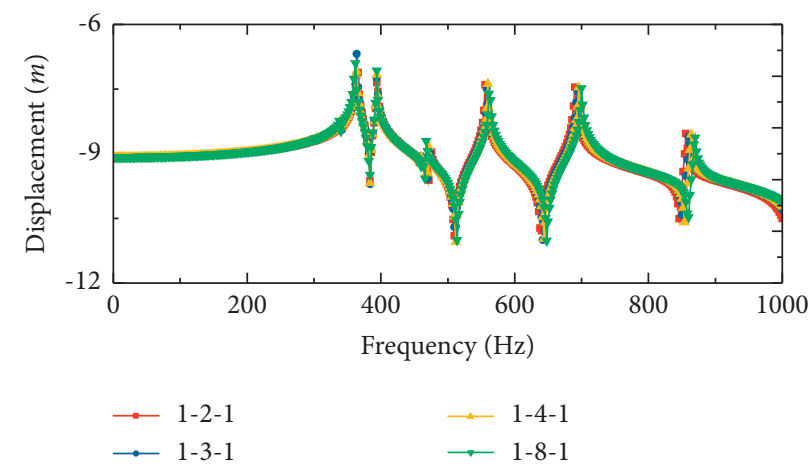

(b)

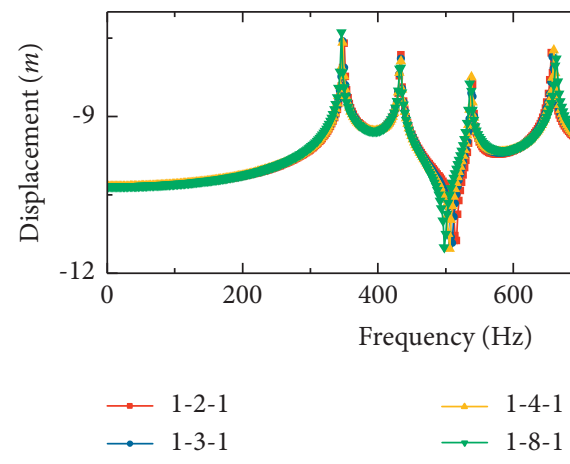

(c)

FIGURE 14: Study on the effect of thickness on the steady-state response of Sandwich FGP doubly curved structure: (a) Sandwich paraboloidal honeycomb shell; (b) Sandwich hyperbolic honeycomb shell; (c) Sandwich elliptical honeycomb shell.

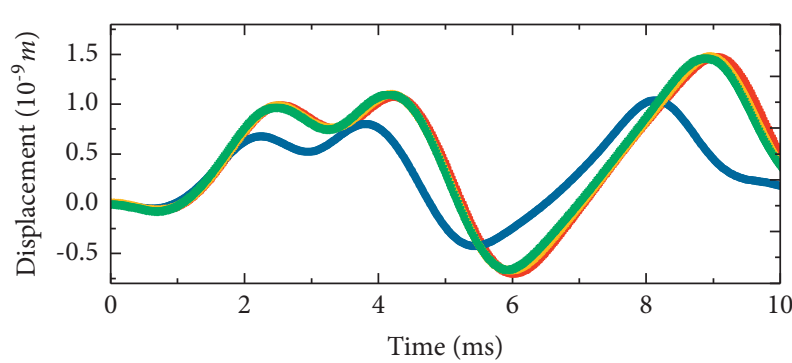

$\rightarrow-1-2-1$
$\rightarrow-1-3-1$
$-1-4-1$

$\rightarrow$ 1-8-1

(a)



$\rightarrow$ 1-2-1

$\rightarrow$ 1-3-1

$-1-4-1$

(b)

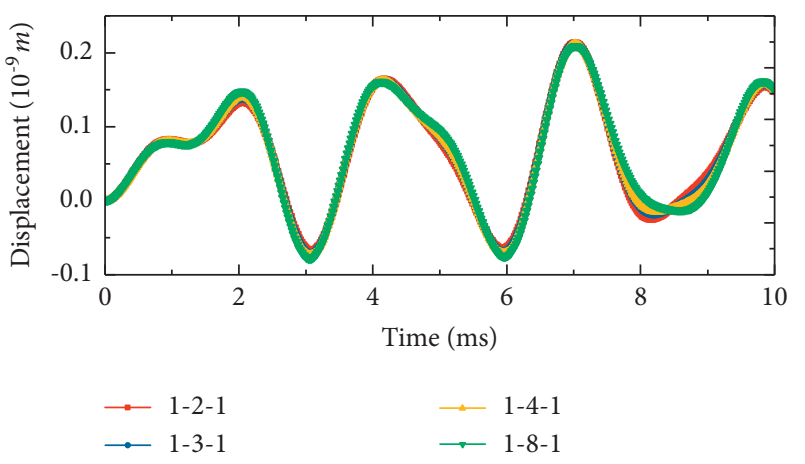

(c)

FIGURE 15: Study on the effect of thickness on the transient response of Sandwich FGP doubly curved structure: (a) Sandwich paraboloidal honeycomb shell; (b) Sandwich hyperbolic honeycomb shell; (c) Sandwich elliptical honeycomb shell. 
resonance peak occurs at larger frequencies as the thickness increases.

\section{Conclusions}

In this research, free and force vibration analyses of a novel Sandwich FGP doubly curved shell subjected to arbitrary boundary conditions are conducted in the framework of the FSDT. The theoretical modeling is based on the domain energy decomposition and boundary spring simulation techniques. For every segment, the displacement functions are represented by Jacobi polynomials along the generatrix orientation and trigonometric series in the circumferential orientation. Free vibration and forced response of Sandwich structures can be obtained by standard variational operation of unknown coefficients of displacement functions. The convergence and validity of the established analysis model are given through several numerical cases. Some new results are given, which may be served as reference data. In addition, parameterized study of forced response is also carried out for pore parameters, boundary parameters, and thickness parameters.

\section{Data Availability}

The data used to support the findings of this study are included within the article.

\section{Conflicts of Interest}

The authors declare that there are no conflicts of interest regarding the publication of this paper.

\section{Acknowledgments}

The authors gratefully acknowledge the financial support from the National Natural Science Foundation of China (Grant no. 51909109) and the Natural Science Foundation of Jiangsu Province (Grant no. BK20190967). The authors also gratefully acknowledge the support from Natural Science Foundation of the Jiangsu High Education Institutions (Grant no. 19KJB580010).

\section{References}

[1] H. Li, F. Pang, Y. Li, and C. Gao, "Application of first-order shear deformation theory for the vibration analysis of functionally graded doubly-curved shells of revolution," Composite Structures, vol. 212, pp. 22-42, 2019.

[2] B. Qin, K. Choe, T. Wang, and Q. Wang, "A unified JacobiRitz formulation for vibration analysis of the stepped coupled structures of doubly-curved shell," Composite Structures, vol. 220, pp. 717-735, 2019.

[3] F. Tornabene, "On the critical speed evaluation of arbitrarily oriented rotating doubly-curved shells made of functionally graded materials," Thin-Walled Structures, vol. 140, pp. 85-98, 2019.

[4] C. Zhang, L. Wang, A. Eyvazian, K. Afrasyab, and T. A. Sebaey, "Analytical solution for static and dynamic analysis of FGP cylinders integrated with FG-GPLs patches exposed to longitudinal magnetic field," Engineering with Computers, pp. 1-19, 2021.

[5] F. Tornabene and M. Bacciocchi, "Dynamic stability of doubly-curved multilayered shells subjected to arbitrarily oriented angular velocities: numerical evaluation of the critical speed," Composite Structures, vol. 201, pp. 1031-1055, 2018.

[6] D. Chen, S. Kitipornchai, and J. Yang, "Dynamic response and energy absorption of functionally graded porous structures," Materials \& Design, vol. 140, pp. 473-487, 2018.

[7] S. Sahmani, M. M. Aghdam, and T. Rabczuk, "Nonlinear bending of functionally graded porous micro/nano-beams reinforced with graphene platelets based upon nonlocal strain gradient theory," Composite Structures, vol. 186, pp. 68-78, 2018.

[8] G.-L. She, F.-G. Yuan, Y.-R. Ren, H.-B. Liu, and W.-S. Xiao, "Nonlinear bending and vibration analysis of functionally graded porous tubes via a nonlocal strain gradient theory," Composite Structures, vol. 203, pp. 614-623, 2018.

[9] D. Wu, A. Liu, Y. Huang, Y. Huang, Y. Pi, and W. Gao, "Dynamic analysis of functionally graded porous structures through finite element analysis," Engineering Structures, vol. 165, pp. 287-301, 2018.

[10] J. Yang, D. Chen, and S. Kitipornchai, "Buckling and free vibration analyses of functionally graded graphene reinforced porous nanocomposite plates based on Chebyshev-Ritz method," Composite Structures, vol. 193, pp. 281-294, 2018.

[11] C. Cui, Z. Wang, W. Zhou, Y. Wu, and W. Wei, "Branch point algorithm for structural irregularity determination of honeycomb," Composites Part B: Engineering, vol. 162, pp. 323330, 2019.

[12] J. Liu, W. Chen, H. Hao, and Z. Wang, "Numerical study of low-speed impact response of sandwich panel with tube filled honeycomb core," Composite Structures, vol. 220, pp. 736$748,2019$.

[13] Z. Wang, Z. Li, and W. Xiong, "Experimental investigation on bending behavior of honeycomb sandwich panel with ceramic tile face-sheet," Composites Part B: Engineering, vol. 164, pp. 280-286, 2019.

[14] Z. Wang, Z. Li, and W. Xiong, "Numerical study on threepoint bending behavior of honeycomb sandwich with ceramic tile," Composites Part B: Engineering, vol. 167, pp. 63-70, 2019.

[15] Z. Wang and J. Liu, "Numerical and theoretical analysis of honeycomb structure filled with circular aluminum tubes subjected to axial compression," Composites Part B: Engineering, vol. 165, pp. 626-635, 2019.

[16] A. M. Zenkour, "A comprehensive analysis of functionally graded sandwich plates: Part 1-Deflection and stresses," International Journal of Solids and Structures, vol. 42, no. 18, pp. 5224-5242, 2005.

[17] A. M. Zenkour, "A comprehensive analysis of functionally graded sandwich plates: Part 2-Buckling and free vibration," International Journal of Solids and Structures, vol. 42, no. 18, pp. 5243-5258, 2005.

[18] S. Natarajan and G. Manickam, "Bending and vibration of functionally graded material sandwich plates using an accurate theory," Finite Elements in Analysis and Design, vol. 57, pp. 32-42, 2012.

[19] A. Mahi, E. A. Adda Bedia, and A. Tounsi, "A new hyperbolic shear deformation theory for bending and free vibration analysis of isotropic, functionally graded, sandwich and laminated composite plates," Applied Mathematical Modelling, vol. 39, no. 9, pp. 2489-2508, 2015. 
[20] C. H. Thai, V. N. V. Do, and H. Nguyen-Xuan, "An improved Moving Kriging-based meshfree method for static, dynamic and buckling analyses of functionally graded isotropic and sandwich plates," Engineering Analysis with Boundary Elements, vol. 64, pp. 122-136, 2016.

[21] D. Xiao, L. Mu, and G. Zhao, "The influence of correlating material parameters of gradient foam core on free vibration of sandwich panel," Composites Part B: Engineering, vol. 77, pp. 153-161, 2015.

[22] M. Liu, Y. Cheng, and J. Liu, "High-order free vibration analysis of sandwich plates with both functionally graded face sheets and functionally graded flexible core," Composites Part B: Engineering, vol. 72, pp. 97-107, 2015.

[23] A. M. Zenkour, "A quasi-3D refined theory for functionally graded single-layered and sandwich plates with porosities," Composite Structures, vol. 201, pp. 38-48, 2018.

[24] S. Pandey and S. Pradyumna, "A layerwise finite element formulation for free vibration analysis of functionally graded sandwich shells," Composite Structures, vol. 133, pp. 438-450, 2015.

[25] H. Chen, A. Wang, Y. Hao, and W. Zhang, "Free vibration of FGM sandwich doubly-curved shallow shell based on a new shear deformation theory with stretching effects," Composite Structures, vol. 179, pp. 50-60, 2017.

[26] M.-C. Trinh and S.-E. Kim, "Nonlinear stability of moderately thick functionally graded sandwich shells with double curvature in thermal environment," Aerospace Science and Technology, vol. 84, pp. 672-685, 2019.

[27] G. Jin, T. Ye, X. Wang, and X. Miao, "A unified solution for the vibration analysis of FGM doubly-curved shells of revolution with arbitrary boundary conditions," Composites Part B: Engineering, vol. 89, pp. 230-252, 2016.

[28] T. Ye, G. Jin, and Y. Zhang, "Vibrations of composite laminated doubly-curved shells of revolution with elastic restraints including shear deformation, rotary inertia and initial curvature," Composite Structures, vol. 133, pp. 202-225, 2015.

[29] H. Li, F. Pang, H. Chen, and Y. Du, "Vibration analysis of functionally graded porous cylindrical shell with arbitrary boundary restraints by using a semi analytical method," Composites Part B: Engineering, vol. 164, pp. 249-264, 2019.

[30] H. Li, F. Pang, X. Miao, S. Gao, and F. Liu, "A semi analytical method for free vibration analysis of composite laminated cylindrical and spherical shells with complex boundary conditions," Thin-Walled Structures, vol. 136, pp. 200-220, 2019.

[31] Z. Qin, X. Pang, B. Safaei, and F. Chu, "Free vibration analysis of rotating functionally graded CNT reinforced composite cylindrical shells with arbitrary boundary conditions," Composite Structures, vol. 220, pp. 847-860, 2019.

[32] K. Choe, J. Tang, C. Shui, A. Wang, and Q. Wang, "Free vibration analysis of coupled functionally graded (FG) doubly-curved revolution shell structures with general boundary conditions," Composite Structures, vol. 194, pp. 413-432, 2018.

[33] K. Choe, Q. Wang, J. Tang, and C. shui, "Vibration analysis for coupled composite laminated axis-symmetric doubly-curved revolution shell structures by unified Jacobi-Ritz method," Composite Structures, vol. 194, pp. 136-157, 2018.

[34] Q. Wang, K. Choe, D. Shi, and K. Sin, "Vibration analysis of the coupled doubly-curved revolution shell structures by using Jacobi-Ritz method," International Journal of $\mathrm{Me}$ chanical Sciences, vol. 135, pp. 517-531, 2018.

[35] J. Zhao, F. Xie, A. Wang, C. Shuai, J. Tang, and Q. Wang, "Vibration behavior of the functionally graded porous (FGP) doubly-curved panels and shells of revolution by using a semi- analytical method," Composites Part B: Engineering, vol. 157, pp. 219-238, 2019.

[36] J. Guo, D. Shi, Q. Wang, J. Tang, and C. Shuai, "Dynamic analysis of laminated doubly-curved shells with general boundary conditions by means of a domain decomposition method," International Journal of Mechanical Sciences, vol. 138-139, pp. 159-186, 2018. 